\title{
An Hybrid Finite Volume-Finite Element Method for VARIABLE DENSITY INCOMPRESSIBLE FLOWS
}

\author{
Caterina Calgaro ${ }^{1,2}$, Emmanuel Creusé ${ }^{1,3, *}$, Thierry Goudon ${ }^{1,2}$ \\ ${ }^{1}$ Equipe-Projet SIMPAF, Centre de Recherche INRIA Futurs \\ Parc Scientifique de la Haute Borne, Avenue Halley B.P. 70478 \\ F-59658 Villeneuve d'Ascq cedex, France. \\ ${ }^{2}$ Laboratoire Paul Painlevé, UMR 8524, \\ C.N.R.S.-Université Sciences et Technologies de Lille, \\ Cité Scientifique, F-59655 Villeneuve d'Ascq cedex, France \\ ${ }^{3}$ Laboratoire Mathématiques et Applications de Valenciennes, FR C.N.R.S. 2956, \\ Université de Valenciennes et du Hainaut-Cambrésis \\ Le Mont Houy, F-59313 Valenciennes cedex 09, France \\ * Corresponding Author \\ E-mails: Caterina.Calgaro@math.univ-lille1.fr, \\ Emmanuel.Creuse@univ-valenciennes.fr, Thierry.Goudon@inria.fr
}

\begin{abstract}
This paper is devoted to the numerical simulation of variable density incompressible flows, modeled by the Navier-Stokes system. We introduce an hybrid scheme which combines a Finite Volume approach for treating the mass conservation equation and a Finite Element method to deal with the momentum equation and the divergence free constraint. The breakthrough relies on the definition of a suitable footbridge between the two methods, through the design of compatibility condition. In turn, the method is very flexible and allows to deal with unstructured meshes. Several numerical tests are performed to show the scheme capabilities. In particular, the viscous Rayleigh-Taylor instability evolution is carefully investigated.
\end{abstract}

Key words. Incompressible Navier-Stokes Equations, Variable density flows, Finite Element method, Finite Volume method, Rayleigh-Taylor instability.

AMS Subject classification. 65M99, 76M10, 76D05, 76M12, 76E17. 


\section{Introduction}

This paper is devoted to the numerical simulation of the variable density incompressible NavierStokes system given on a domain $\Omega \subset \mathbb{R}^{N}$ by :

$$
\begin{aligned}
\partial_{t} \rho+\operatorname{div}_{\mathbf{x}}(\rho \mathbf{u}) & =0 \\
\partial_{t}(\rho \mathbf{u})+\operatorname{Div}_{\mathbf{x}}(\rho \mathbf{u} \otimes \mathbf{u})+\nabla_{\mathbf{x}} p-\mu \Delta_{\mathbf{x}} \mathbf{u} & =\mathbf{f}, \\
\operatorname{div}_{\mathbf{x}} \mathbf{u} & =0 .
\end{aligned}
$$

Here $\rho(t, \mathbf{x}) \geq 0$ stands for the density of a viscous fluid whose velocity field is $\mathbf{u}(t, \mathbf{x}) \in \mathbb{R}^{N}$. The description of the external force is embodied into the right hand side $\mathbf{f}(t, \mathbf{x})$ of $(1.2)$ and $\mu>0$ is the viscosity of the fluid. The unknowns depend on time $t \geq 0$ and position $\mathbf{x} \in \Omega \subset \mathbb{R}^{N}$. Given vector fields $\mathbf{u}$ and $\mathbf{v}$ we set $\operatorname{div}_{\mathbf{x}}(\mathbf{u})=\sum_{i=1}^{N} \partial_{x_{i}} u_{i}$, and $\mathbf{u} \otimes \mathbf{v}$ is the $N \times N$ matrix with components $u_{i} v_{j}$; given a matrix valued function $A$ we denote $\operatorname{Div}_{\mathbf{x}} A$ the vector having components $\sum_{j=1}^{N} \partial_{x_{j}} A_{i j}$. The third unknown of the problem is the pressure $p(t, \mathbf{x}) \in \mathbb{R}$; it can be seen as a Lagrange multiplier associated to the incompressibility constraint (1.3). The problem is completed by initial and boundary conditions, to which we shall go back later on.

Neglecting any technical difficulties, the system can be rewritten in many different ways, that have each their own interest both on theoretical or numerical viewpoint. To start with, using (1.3), the mass conservation relation (1.1) can be recast as

$$
\partial_{t} \rho+\mathbf{u} \cdot \nabla_{\mathbf{x}} \rho=0 .
$$

Accordingly the density remains constant along the characteristic curves associated to the velocity field $\mathbf{u}$, and therefore, if the density is initially homogeneous $\rho(t=0, \mathbf{x})=\bar{\rho}>0$, then it remains constant for any positive time: $\rho(t, \mathbf{x})=\bar{\rho}$. In this very specific situation the problem reduces to

$$
\left\{\begin{array}{l}
\partial_{t} \mathbf{u}+\left(\mathbf{u} \cdot \nabla_{\mathbf{x}}\right) \mathbf{u}+\frac{1}{\bar{\rho}} \nabla_{\mathbf{x}} p-\frac{\mu}{\bar{\rho}} \Delta_{\mathbf{x}} \mathbf{u}=\frac{1}{\bar{\rho}} \mathbf{f} \\
\operatorname{div}_{\mathbf{x}} \mathbf{u}=0
\end{array}\right.
$$

where we have used (1.3) to develop $\operatorname{Div}_{\mathbf{x}}(\mathbf{u} \otimes \mathbf{u})=\left(\mathbf{u} \cdot \nabla_{\mathbf{x}}\right) \mathbf{u}$. The homogeneous Navier-Stokes system (1.5) has motivated a lot of work since the seminal results of Leray [Ler34]; we refer e.g. to the treatise [Tem01] and for numerical methods to [GR86]; a survey of recent theoretical developments can be found in [Che04]. However, many applications require to deal with inhomogeneous flows, and to consider the full system (1.1), (1.2), (1.3). We just keep in mind that a basic requirement for any scheme dealing with the variable density case is to be able to recover results known for the homogeneous situation.

In the variable density case, the momentum equation (1.2) can also be written in many different ways. As said above, by using (1.1) we obtain

$$
\rho\left(\partial_{t} \mathbf{u}+\left(\mathbf{u} \cdot \nabla_{\mathbf{x}}\right) \mathbf{u}\right)+\nabla_{\mathbf{x}} p-\mu \Delta_{\mathbf{x}} \mathbf{u}=\mathbf{f},
$$

but considering now possibly space varying density. A useful trick consists in writing alternatively

$$
\partial_{t}(\sqrt{\rho} \mathbf{u})+\operatorname{Div}_{\mathbf{x}}(\sqrt{\rho} \mathbf{u} \otimes \mathbf{u})+\frac{1}{\sqrt{\rho}} \nabla_{\mathbf{x}} p-\frac{\mu}{\sqrt{\rho}} \Delta_{\mathbf{x}} \mathbf{u}=\frac{\mathbf{f}}{\sqrt{\rho}} .
$$


This formulation — which is used in [Lio96] and for numerical purposes in [GQ00] — is very convenient since it makes naturally appear the kinetic energy of the fluid which is the $L^{2}$ norm of the quantity $\sqrt{\rho} \mathbf{u}$. Finally, we can also express the divergence free condition (1.3) as a Poisson equation, with variable coefficients, for the pressure

$$
\operatorname{div}_{\mathbf{x}}\left(\frac{1}{\rho} \nabla_{\mathbf{x}} p\right)=\operatorname{div}_{\mathbf{x}}\left(\frac{\mathbf{f}}{\rho}-\left(\mathbf{u} \cdot \nabla_{\mathbf{x}}\right) \mathbf{u}+\frac{\mu}{\rho} \Delta_{\mathbf{x}} \mathbf{u}\right) .
$$

Combined with a discussion of the boundary condition for the pressure $p$, this formulation is the basis of the numerical scheme used in [JL04].

For details on existence results for the non homogeneous incompressible Navier-Stokes system we refer to [Lio96, BF06, Dan04, IT99]. Let us now make a brief overview of the numerical methods used to deal with (1.1)-(1.2)-(1.3). The problem combines the difficulty of the transport equation (1.1) with the difficulty of guaranteeing the divergence free constraint (1.3) in the evolution of the velocity (1.2). A first possible strategy adopts in some sense a compressible and hyperbolic viewpoint: the viscous term is seen as a correction, that certainly helps, and the method relies on transport characteristic based schemes for the unknowns $(\rho, \mathbf{u})$ which are advected by the same velocity field $\mathbf{u}$. Then, the divergence free constraint can be treated by using a projection step $\left[\mathrm{ABC}^{+} 98\right]$ or a fictitious time, see [SG88, GS89]. We also refer to [SD06, SD05a, SD05b]. The second viewpoint, which is closer to the strategy we adopt, is more based on an incompressible philosophy and exploit the viscous term in (1.2). This leads to use modern Finite Elements (FE) methods, see [EG04]. The method uses a time splitting, solving separately the transport equation for the density (1.1) and the momentum equation (1.2), the constraint (1.3) being treated through a projection method, see [GMS06]. This is the methodology followed in [GQ00, FGQ01]. These contributions use the formulation (1.7) since a specific care is given to the kinetic energy relation. The (unconditional) stability of the scheme however requires two projection steps, which is time consuming since one has to solve elliptic equation with variable coefficients (both in time and space). The method has been improved in [PS07] by getting rid of one projection step. Note also that [PS07] found some advantage in coming back to the formulation (1.6). Of course, a difficulty arises with the transport equation (1.1) which, being of hyperbolic nature, is not well adapted to a mere treatment by FE methods, but instead requires a specific approach, like Discontinuous Galerkin methods, artificial viscosity, sub-grid stabilization procedure as in [GQ00], see also [Gue99], or the least-square method as used in [PS07]. Finally, it is worth mentioning [JL04] which designs a Finite Difference scheme for solving the system (1.4), (1.6) and (1.8), completed by a deep discussion of the boundary condition to be used for the pressure. The transport equation is then evaluated by means of a shock capturing method involving slope limiters.

The originality of our work is to use different numerical methods for the transport equation (1.1) and for evaluating the evolution of the velocity driven by (1.2), (1.3). To be more specific, we use a time-splitting, solving (1.1) for a given velocity by using a Finite Volume (FV) approach which is efficient when dealing with a pure convection equation, see [LeV02, EGH00] and then, we compute the divergence free solution of (1.6) by exploiting the advantages of FE methods, see [Tem01, GR86, EG04]. However, the difficulty relies on the compatibility of the two approaches. This question has been addressed for different purposes in [PG01, GP99]. Here, we care to preserve 
the divergence free constraint between the two steps of the splitting. Indeed, for any node $A$, the FE computation gives a $\mathbf{u}$ which satisfies

$$
\int_{\Omega} \operatorname{div}_{\mathbf{x}} \mathbf{u} \psi_{A} \mathrm{~d} \mathbf{x}=0
$$

where $\psi_{A}$ is the FE basis function associated to the node $A$. Then, from this quantity we are left with the task of defining some $\mathbf{u}^{\star}$ on the interface $\partial \mathcal{C}_{A}$ of the control volume $\mathcal{C}_{A}$ associated to the node $A$ in the FV method so that

$$
\int_{\partial \mathcal{C}_{A}} \mathbf{u}^{\star} \cdot \mathbf{n} \mathrm{d} \gamma(\mathbf{x})=0
$$

with $\mathbf{n}$ the outward normal to $\mathcal{C}_{A}$. A naive choice would not satisfy this constraint and as a consequence is not able to give a correct solution in the purely homogeneous case (1.5): since the divergence free condition is not numerically fulfilled (1.1) and (1.4) are not equivalent and spurious space variation of the density appears. In what follows we shall use a FE method based on the Uzawa algorithm for the momentum equation, with $\mathbb{P} 2 / \mathbb{P} 1$ elements. However, we point out that this choice is not crucial and any efficient incompressible method can be used as well, for instance using projection steps as in [GQ00], see also [GMS06], or using a different family of FE. We also warn the reader that many technical choices made below are dictated by the fact that we have in mind to adapt our method to deal with Low Mach number models, as arising in combustion problems, where the divergence free condition is replaced by a relation between $\operatorname{div}_{\mathbf{x}} \mathbf{u}$ and non linear derivatives of the density, see [Lio98] and the numerical investigation in [TSG ${ }^{+}$06, GST04]. Such problems will be discussed elsewhere [CCGS]. There are many advantages in using such an hybrid FV/FE approach. First of all, we can develop independently performing methods well suited to both equations (1.1) and (1.6), (1.3). For instance shock capturing methods and slope limiters can be incorporated easily in the evaluation of the density and many different FE can be used for the velocity. Second of all, the method is very flexible and allows easily the use of unstructured meshes through the design of suitable compatibility conditions. This aspect is discussed in detail in Section 2.4. Definitely, this is a strong motivation for this work. Note also that the method might be advantageously combined with mesh refinements strategies.

The paper is organized as follows. In Section 2 we describe the scheme: we start with a detailed presentation of the method used in each step of the splitting and then we discuss precisely the compatibility conditions. Section 3 is devoted to numerical results: having validated the scheme and discussed experimentally rates of convergence, we offer some examples of non homogeneous flows.

\section{Description of the Numerical Scheme}

The complete mathematical statement of the problem (1.1)-(1.6)-(1.3) requires suitable boundary and initial conditions. Let $\Omega$ be an open bounded set of $\mathbb{R}^{N}$, with a locally Lipschitz boundary $\partial \Omega$; $\mathbf{n}(\mathbf{x})$ denote the outer unit normal vector at $\mathbf{x} \in \partial \Omega$. The Navier-Stokes system is completed by 
imposing

$$
\begin{cases}\mathbf{u}_{\mid \partial \Omega}=\mathbf{g}, & \mathbf{u}_{\left.\right|_{t=0}}=\mathbf{u}_{0}, \\ \rho_{\left.\right|_{\Gamma_{\text {inc }}}}=\rho_{\text {inc }}, & \rho_{\left.\right|_{t=0}}=\rho_{0},\end{cases}
$$

where $\mathbf{g}$ and $\rho_{\text {inc }}>0$ are respectively the velocity and the density prescribed on the boundary, whereas $\mathbf{u}_{0}$ and $\rho_{0}$ are the initial velocity and density. According to the hyperbolic character of the mass conservation equation (1.1), we write

$$
\Gamma_{\text {inc }}=\{\mathbf{x} \in \partial \Omega \mid \mathbf{g}(\mathbf{x}) \cdot \mathbf{n}<0\} .
$$

As for the homogeneous Navier-Stokes problem, neither a boundary condition nor an initial condition is required for the pressure. Finally, the problem is well posed when the following compatibility conditions are satisfied

$$
\int_{\partial \Omega} \mathbf{g} \cdot \mathbf{n} \mathrm{d} \gamma(\mathbf{x})=0 \quad \forall t \geq 0 \quad \text { and } \operatorname{div}_{\mathbf{x}} \mathbf{u}_{0}=0
$$

It is classical that the boundary condition can be transformed into a forcing term so that, without loss of generality, we assume in what follows $\mathbf{g}=0$.

In the literature, FV schemes are widely used in the numerical solution of conservation laws such as (1.1), [LeV02, EGH00]. FE approximations are naturally well suited to elliptic or parabolic i.e. diffusive problems such as (1.6)-(1.3), [BF91, EG04]. The idea of combine FE methods and FV methods in computational fluid dynamics was used in [Pas03, PG01] (see also the reference therein) in order to approximate the solution of convection-diffusion equations. Their aim was to introduce several footbridges between FV and FE for coupling the discretizations of convective and diffusive terms of a system of conservation laws.

In order to couple the advantages of $\mathrm{FV}$ method for the transport equation satisfied by the density and FE method for evaluating the evolution of the velocity and pressure, we consider the time splitting of the Cauchy problem described below. To be more specific, from now on we restrict ourselves to the 2-dimensional framework: $N=2$.

\subsection{The time splitting}

Let us denote $\Delta t$ the time step and $t^{n}=n \Delta t, n \geq 0$. Let us assume that the numerical solution at time $t^{n}$, namely $\left(\rho^{n}, \mathbf{u}^{n}, p^{n}\right)$, is known on the computational domain. The time splitting of the system (1.1)-(1.6)-(1.3) is known as the "Strang splitting" [Str68] :

1. The new density field, $\rho^{n+1}$, is computed by solving on the time interval $(n \Delta t,(n+1) \Delta t)$ the transport equation

$$
\partial_{t} \rho^{n+1}+\operatorname{div}_{\mathbf{x}}\left(\rho^{n+1} \mathbf{u}^{n}\right)=0,
$$

with suitable boundary conditions on $\rho^{n+1}$. Details of the method we use are given in Section 2.3 . 
2. The new velocity and pressure fields, $\mathbf{u}^{n+1}$ and $p^{n+1}$, are computed by the resolution on the time interval $(n \Delta t,(n+1) \Delta t)$ of the system

$$
\begin{array}{r}
\rho^{n+1}\left(\partial_{t} \mathbf{u}^{n+1}+\mathbf{u}^{n+1} \cdot \nabla_{\mathbf{x}} \mathbf{u}^{n+1}\right)+\nabla_{\mathbf{x}} p^{n+1}-\mu \Delta_{\mathbf{x}} \mathbf{u}^{n+1}=\mathbf{f}^{n+1}, \\
\operatorname{div}_{\mathbf{x}} \mathbf{u}^{n+1}=0,
\end{array}
$$

completed by the specification of boundary conditions on $\mathbf{u}^{n+1}$. Details of the method we use are given in Section 2.2.

3. The following velocity and pressure fields, $\mathbf{u}^{n+2}$ and $p^{n+2}$, are computed by the resolution on the time interval $((n+1) \Delta t,(n+2) \Delta t)$ of the system

$$
\begin{array}{r}
\rho^{n+1}\left(\partial_{t} \mathbf{u}^{n+2}+\mathbf{u}^{n+2} \cdot \nabla_{\mathbf{x}} \mathbf{u}^{n+2}\right)+\nabla_{\mathbf{x}} p^{n+2}-\mu \Delta_{\mathbf{x}} \mathbf{u}^{n+2}=\mathbf{f}^{n+2}, \\
\operatorname{div}_{\mathbf{x}} \mathbf{u}^{n+2}=0,
\end{array}
$$

completed by the specification of boundary conditions on $\mathbf{u}^{n+2}$.

4. Finally, the new density field, $\rho^{n+2}$, is computed by solving on the time interval $((n+1) \Delta t,(n+$ 2) $\Delta t$ ) the transport equation

$$
\partial_{t} \rho^{n+2}+\operatorname{div}_{\mathbf{x}}\left(\rho^{n+2} \mathbf{u}^{n+2}\right)=0,
$$

with suitable boundary conditions on $\rho^{n+2}$.

Then we go back to the first step (using $n+2$ instead of $n$ ) to compute the solution at the following time steps.

\subsection{Solving the Velocity Equation by a FE Method}

In the numerical simulation of the Navier-Stokes equations (2.10), a major difficulty is that the velocity and the pressure are coupled by the incompressibility constraint (2.11). From the seminal works of Chorin and Temam [Tem68, Cho68], the interest in using projection methods is that, at each time step, one only needs to solve a sequence of decoupled elliptic equations for the velocity and the pressure. One can see [GMS06] and references therein for an overview of projection methods

for incompressible flows. Here, we shall use instead the Uzawa algorithm which is based on a saddle-point interpretation of the problem, see [Tem01, Tur99]. In a future work, our goal is to generalize the present scheme to low Mach number models [CCGS], where the constraint (1.3) will be replaced by

$$
\operatorname{div}_{\mathbf{x}} \mathbf{u}=\Delta_{\mathbf{x}} F(\rho),
$$

for a certain function $F: \mathbb{R} \rightarrow \mathbb{R}$. The classical Uzawa algorithm is still well adapted to treat such a situation. Indeed, when the homogeneous, or non homogeneous, Navier-Stokes equations are discretized by a FE method, the resulting saddle-point problem can be put into the framework of an optimization problem and can be solved with general methods of descent as Uzawa's algorithm, penalty method, augmented Lagrangian algorithm (see [BF91]) or stabilized saddle-point problem (see [CDJ06] and reference therein). 
Since we aim at using a FE method, it is convenient to write the variational formulation of (2.10)-(2.11). As usual we denote by $L_{0}^{2}(\Omega)$ the space of square integrable functions having a vanishing mean over $\Omega$. Let $\rho \geq 0$ be a given function defined on $\Omega$, say $\rho \in L^{1} \cap L^{\infty}(\Omega)$. We aim at solving

$$
\left\{\begin{array}{c}
\text { Find }(\mathbf{u}, p) \in\left(H_{0}^{1}(\Omega)\right)^{2} \times L_{0}^{2}(\Omega) \text { such that for any }(\mathbf{v}, q) \in\left(H_{0}^{1}(\Omega)\right)^{2} \times L_{0}^{2}(\Omega) \\
\left(\rho \partial_{t} \mathbf{u}, \mathbf{v}\right)+b(\rho \mathbf{u}, \mathbf{u}, \mathbf{v})+a(\mathbf{u}, \mathbf{v})+d(\mathbf{v}, p)=(\mathbf{f}, \mathbf{v}) \\
d(\mathbf{u}, q)=0
\end{array}\right.
$$

on the time step $(n \Delta t,(n+1) \Delta t)$, with data $\mathbf{u}_{\mid t=n \Delta t}=\mathbf{u}^{n}$. Here, $(.,$.$) is the usual L^{2}(\Omega)$ (or $\left.\left(L^{2}(\Omega)\right)^{2}\right)$ inner product, $a(.,$.$) and d(.,$.$) are the bilinear forms defined by:$

$$
\begin{array}{ll}
a(\mathbf{u}, \mathbf{v})=\mu\left(\nabla_{\mathbf{x}} \mathbf{u}, \nabla_{\mathbf{x}} \mathbf{v}\right), & \mathbf{u}, \mathbf{v} \in\left(H_{0}^{1}(\Omega)\right)^{2}, \\
d(\mathbf{v}, p)=-\left(p, \operatorname{div}_{\mathbf{x}} \mathbf{v}\right), & \mathbf{v} \in\left(H_{0}^{1}(\Omega)\right)^{2}, p \in L_{0}^{2}(\Omega),
\end{array}
$$

and $b(., .,$.$) is the trilinear form defined by$

$$
b(\mathbf{u}, \mathbf{v}, \mathbf{w})=\left(\left(\mathbf{u} \cdot \nabla_{\mathbf{x}}\right) \mathbf{v}, \mathbf{w}\right), \quad \mathbf{u}, \mathbf{v}, \mathbf{w} \in\left(H_{0}^{1}(\Omega)\right)^{2} .
$$

Of course, $a(.,$.$) is elliptic on \left(H_{0}^{1}(\Omega)\right)^{2}$ and $d(.,$.$) satisfies the Babuska-Brezzi "inf-sup" condition$ on $\left(H_{0}^{1}(\Omega)\right)^{2} \times L_{0}^{2}(\Omega)$. Now let us discuss the discrete version of $(2.16)$.

The domain $\Omega$ is approximated by a computational domain $\Omega_{h}$, discretized by a conforming and isotropic set of triangles $\mathcal{T}_{h}$, with mesh-size $h$. Let us introduce FE spaces $V_{h} \subset\left(H_{0}^{1}\left(\Omega_{h}\right)\right)^{2}$ for the velocity $\mathbf{u}_{h}$ and $Q_{h} \subset L_{0}^{2}\left(\Omega_{h}\right)$ for the pressure $p_{h}$. It is well know that, when choosing a pair of FE spaces $V_{h}, Q_{h}$ to discretize the velocity and the pressure, it is necessary to check the Babuska-Brezzi "inf-sup" condition. We define

$$
\begin{array}{r}
V_{h}=\left\{\mathbf{v}_{h} \in \mathcal{C}^{0}\left(\bar{\Omega}_{h}\right) \mid \mathbf{v}_{\left.h\right|_{K}} \in \mathcal{Q}(K) \forall K \in \mathcal{T}_{h}\right\}, \\
Q_{h}=\left\{q_{h} \in \mathcal{C}^{0}\left(\bar{\Omega}_{h}\right) \mid q_{\left.\right|_{K}} \in \mathcal{P}(K) \forall K \in \mathcal{T}_{h}\right\}
\end{array}
$$

where for all $K \in \mathcal{T}_{h}, \mathcal{P}(K)$ and $\mathcal{Q}(K)$ are spaces of polynomials with degree $p$ and $q$, respectively. For our simulations, we choose $\mathcal{P}(K)=\mathbb{P}_{1}$ and $\mathcal{Q}(K)=\mathbb{P}_{2}$. Among the possible choices, we have eliminated elements when the pressure is piecewise constant. Indeed, it seems that such discretizations produce oscillations when highly non stationary flows are computed [Cal96]. Another possibility is the so-called mini-element (or $\mathbb{P}_{1}$-bubble $\mathbb{P}_{1}$ ), which can be seen as a stabilized $\mathbb{P}_{1}-\mathbb{P}_{1}$ FE [CDJ06]. This choice produces a lower approximation of the velocity field but it reduces the number of degrees of freedom in comparison to stable discretizations, which will be essential for 3D problems. The non-conforming FE for the pressure are also possible, in particularly when the domain $\Omega_{h}$ is not convex.

Now, we also need to define a suitable discrete approximation of the (given) function $\rho$, compatible with the discretization of the velocity and the pressure. This means that we need a finite dimensional space $Y_{h}$, based on piecewise polynomial functions, and $\rho_{h} \in Y_{h}$ approximating $\rho$. Since the formulation (2.16) does not involve more regularity assumption on $\rho$ than on the pressure $p$, a natural choice is merely $Y_{h}=Q_{h}$. Moreover, if we consider a piecewise constant density, we 
cannot extend the present work to the modified incompressible equation (2.15). We keep in mind that actually $\rho$ comes from a FV approximation of the density and it is therefore defined on control volumes associated to a dual mesh. We shall detail in Section 2.4 below the construction of the approximation belonging to the FE space.

Let us conclude with a few words about the time discretization of (2.16). We shall use a semiimplicit scheme to treat the nonlinear convection term, as a result of a linearization step in the momentum equation (1.6). This scheme, already used in [GQ00], is a second-order accuracy in time. Precisely, given the approximations $\mathbf{u}^{n}, \mathbf{u}^{n-1}$ and $\rho^{*}=\rho^{n+1}\left(\operatorname{resp} . \rho^{*}=\rho^{n}\right.$ ) in the second (resp. third) step of the Strang splitting, at time $t^{n+1}$ we compute $\left(\mathbf{u}^{n+1}, p^{n+1}\right)$ by solving

$$
\begin{aligned}
\rho^{*}\left(\frac{\left(3 \mathbf{u}^{n+1}-4 \mathbf{u}^{n}+\mathbf{u}^{n-1}\right)}{2 \Delta t}+\left(\overline{\mathbf{u}}^{n+1} \cdot \nabla_{\mathbf{x}}\right) \mathbf{u}^{n+1}\right)-\mu \Delta_{\mathbf{x}} \mathbf{u}^{n+1}+\nabla_{\mathbf{x}} p^{n+1} & =\mathbf{f}^{n+1} \\
\operatorname{div}_{\mathbf{x}} \mathbf{u}^{n+1} & =0
\end{aligned}
$$

Here, $\overline{\mathbf{u}}^{n+1}=2 \mathbf{u}^{n}-\mathbf{u}^{n-1}$ is the linear second-order extrapolation of the velocity field at the new time $t^{n+1}$. Obviously, other schemes can also be considered, in which the nonlinear term $\left(\mathbf{u} \cdot \nabla_{\mathbf{x}}\right) \mathbf{u}$ can appear as a forcing term, if it is treated in a a fully explicit way.

\subsection{Solving the Density Equation by a FV Method}

Let $\mathbf{u}$ be a given velocity field defined on $\Omega$, with $\mathbf{u} \in H_{0}^{1}(\Omega)$. We aim at solving the transport equation

$$
\partial_{t} \rho+\operatorname{div}_{\mathbf{x}}(\rho \mathbf{u})=0
$$

on the time step $(n \Delta t,(n+1) \Delta t)$, with data $\rho_{\mid t=n \Delta t}=\rho^{n}$. The computation of the solution is performed using a usual vertex-based FV scheme. Without loss of generality, let us describe how the value of $\rho_{A}$ is obtained, where $A$ is an internal node of the triangulation, see Figure 1.

\subsubsection{Mesh Definitions}

Let us suppose that $A$ is the common vertex of $n t$ triangles (see Figure 1 for the case $n t=5$ ). Here $A$ is an internal point which means $A$ belongs to $\Omega$ and we shall detail the case of a point of the boundary in a specific section later on. We denote $A_{i}$ the neighboring nodes of $A$, enumerated counterclockwise and $M_{i}$ the triangle $\left(A, A_{i}, A_{i+1}\right), 1 \leq i \leq n t$. We also denote $A_{i, i+1}$ the middle of $\left[A_{i} A_{i+1}\right], A_{i}^{\prime}$ the middle of $\left[A A_{i}\right]$ and $A_{i, i+1}^{\prime}$ the isobarycenter of $\left\{A, A_{i}, A_{i+1}\right\}(1 \leq i \leq n t)$. By convention, an index value $i$ must be understood as $(i \bmod n t)$ (for example, $A_{n t+1}$ is the same as $A_{1}$ or $A_{n t, n t+1}$ is the same as $A_{n t, 1}$ ), and the indices can commute (for example, $A_{n t, 1}$ is the same as $\left.A_{1, n t}\right)$. We define the vertex-based control volume $\mathcal{C}_{A}$ associated to the node $A$, by joining all the nodes $A_{i}^{\prime}$ and $A_{i, i+1}^{\prime}$ for $1 \leq i \leq n t$ (see Figure 1 ). The boundary $\partial \mathcal{C}_{A}$ of $\mathcal{C}_{A}$ splits in several parts $\Gamma_{i}^{-}$and $\Gamma_{i}^{+}$, where $\Gamma_{i}^{-}=\left[A_{i-1, i}^{\prime} A_{i}^{\prime}\right]$ and $\Gamma_{i}^{+}=\left[A_{i}^{\prime} A_{i, i+1}^{\prime}\right](2 \leq i \leq n t+1)$. The approximated density $\rho_{A}$ is built as a constant function on $\mathcal{C}_{A}$. Let us remark that the cell-vertex choice of the FV method (instead of the cell-center one) allows to write a $\mathbb{P}_{1}$ Lagrange interpolation of the density if needed, by using this constant value $\rho_{A}$ on $\mathcal{C}_{A}$ as the value of $\rho$ at node $A$ (see section 2.4). Let $\left|\mathcal{C}_{A}\right|$ stand for the area of $\mathcal{C}_{A},\left|M_{i}\right|$ for the area of $M_{i}$, and $\left|\Gamma_{i}^{-}\right|$(resp. $\left.\left|\Gamma_{i}^{+}\right|\right)$is the length of $\Gamma_{i}^{-}$ 


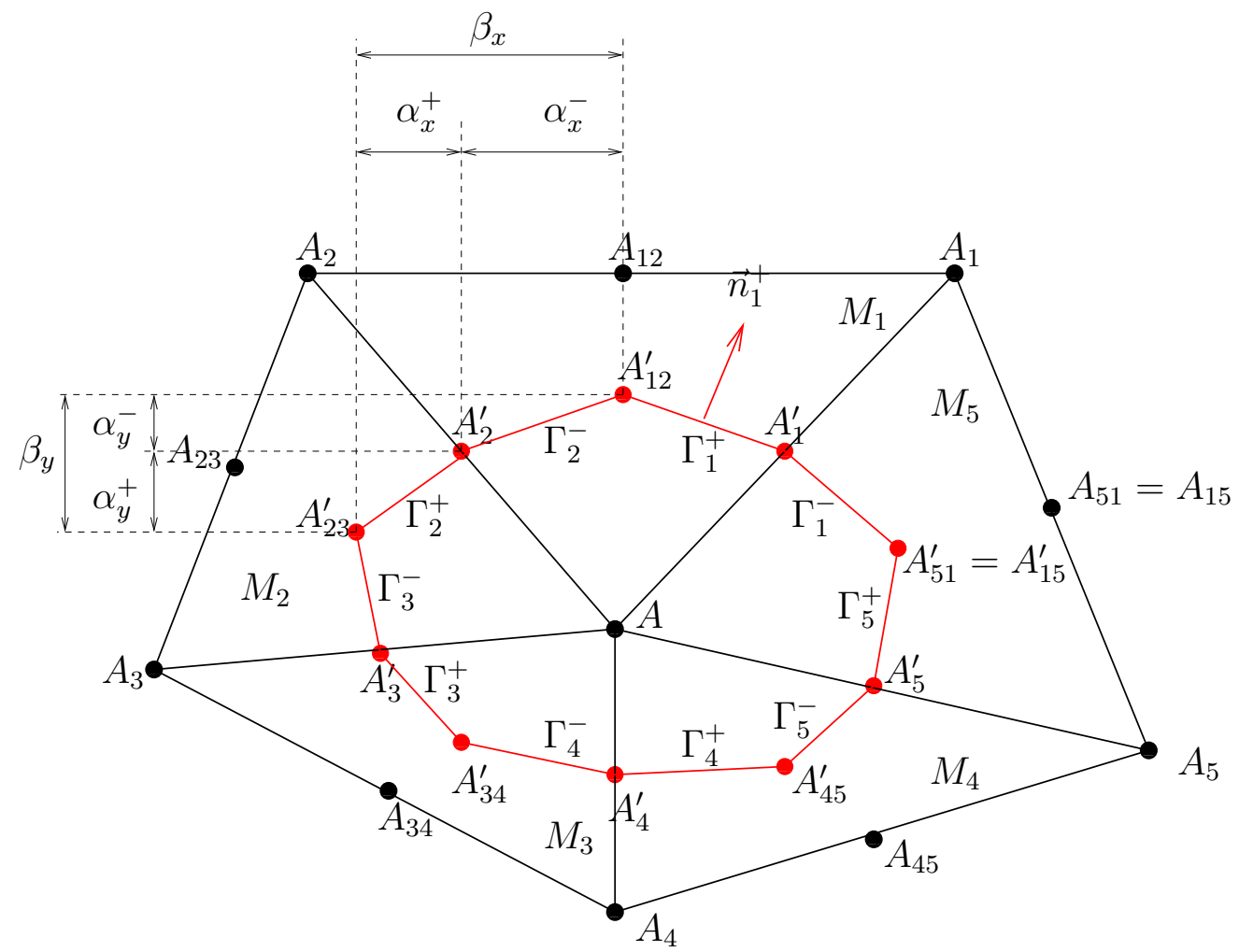

Figure 1: Node $A$ on unstructured mesh for $n t=5$. 
(resp. $\Gamma_{i}^{+}$). Finally, $\mathbf{n}_{i}^{-}$(resp. $\mathbf{n}_{i}^{+}$) is the unit outward normal to $\mathcal{C}_{A}$ along $\Gamma_{i}^{-}$(resp. $\Gamma_{i}^{+}$). The coordinates of $A$ are denoted $(x, y)$ and the coordinates of $A_{i}$ are denoted $\left(x_{i}, y_{i}\right), 1 \leq i \leq n t$. For a given function $f: \Omega \rightarrow \mathbb{R}$, the shorthand notation $f, f_{i}, f_{i}^{\prime}, f_{i, j}$ and $f_{i, j}^{\prime}$ stand for $f(A), f\left(A_{i}\right)$, $f\left(A_{i}^{\prime}\right), f\left(A_{i, j}\right)$ and $f\left(A_{i, j}^{\prime}\right)$, respectively.

\subsubsection{The Finite Volume Scheme}

Integrating $(2.9)$ on $\mathcal{C}_{A}$ yields

$$
\int_{\mathcal{C}_{A}} \partial_{t} \rho \mathrm{d} \mathbf{x}+\int_{\mathcal{C}_{A}} \operatorname{div}_{\mathbf{x}}(\rho \mathbf{u}) \mathrm{d} \mathbf{x}=0
$$

and using the Green formula, we get

$$
\int_{\mathcal{C}_{A}} \partial_{t} \rho \mathrm{d} \mathbf{x}+\sum_{i=1}^{n t}\left(\int_{\Gamma_{i}^{-}} \rho \mathbf{u} \cdot \mathbf{n}_{i}^{-} \mathrm{d} \gamma(\mathbf{x})+\int_{\Gamma_{i}^{+}} \rho \mathbf{u} \cdot \mathbf{n}_{i}^{+} \mathrm{d} \gamma(\mathbf{x})\right)=0 .
$$

The numerical approximation of the density $\rho$ is based on the following ingredients:

- We define the approximation as to be constant on $\mathcal{C}_{A}$, denoting by $\rho_{A}$ the value on the volume control;

- Knowing $\mathbf{u}$ on the whole domain $\Omega$, we introduce a auxiliary velocity $\mathbf{u}^{\star}$ on $\partial \mathcal{C}_{A}$, which is constant on each set $\Gamma_{i}^{-}$and $\Gamma_{i}^{+}$. Namely, we construct $\mathbf{u}^{\star}$ such that

$$
\text { For any } x \in \partial \mathcal{C}_{A}=\bigcup_{i=1}^{n t}\left(\Gamma_{i}^{+} \cup \Gamma_{i}^{-}\right), \mathbf{u}^{\star}(\mathbf{x})= \begin{cases}\mathbf{u}_{i}^{\star-} & \text { on } \Gamma_{i}^{-}, \\ \mathbf{u}_{i}^{\star+} & \text { on } \Gamma_{i}^{+} .\end{cases}
$$

where the values $\mathbf{u}_{i}^{\star \pm}$ will depend on the original velocity $\mathbf{u}$, see Section 2.4 below. Then, we compute $\rho_{A}$ by coming back to (2.19) which yields

$$
\partial_{t} \rho_{A}=-\frac{1}{\left|\mathcal{C}_{A}\right|} \sum_{i=1}^{n t}\left(\mathbf{u}_{i}^{\star-} \cdot \mathbf{n}_{i}^{-} \int_{\Gamma_{i}^{-}} \rho \mathrm{d} \gamma(\mathbf{x})+\mathbf{u}_{i}^{\star+} \cdot \mathbf{n}_{i}^{+} \int_{\Gamma_{i}^{+}} \rho \mathrm{d} \gamma(\mathbf{x})\right) .
$$

Knowing the value $\rho_{A} \mid t=n \Delta t=\rho_{A}^{n}$, we compute the value of $\rho_{A}^{n+1}$ by using a fully explicit second order Runge-Kutta integration scheme. At each step of this integration method, we have to evaluate the right hand side of $(2.20)$. Having at hand two interpolation values $\widetilde{\rho}_{A}^{+}$and $\widetilde{\rho}_{A_{i}}^{+}$(respectively $\widetilde{\rho}_{A}^{-}$ and $\widetilde{\rho}_{A_{i}}^{-}$) for $\rho$ on $\Gamma_{i}^{+}$(respectively $\Gamma_{i}^{-}$), we set

$$
\begin{gathered}
\int_{\Gamma_{i}^{+}} \rho \mathrm{d} \gamma(\mathbf{x})= \begin{cases}\left|\Gamma_{i}^{+}\right| \widetilde{\rho}_{A_{i}}^{+} & \text {if } \mathbf{u}_{i}^{\star+} \cdot \mathbf{n}_{i}^{-} \leq 0, \\
\left|\Gamma_{i}^{+}\right| \widetilde{\rho}_{A}^{+} & \text {if } \mathbf{u}_{i}^{\star+} \cdot \mathbf{n}_{i}^{-}>0,\end{cases} \\
\int_{\Gamma_{i}^{-}} \rho \mathrm{d} \gamma(\mathbf{x})= \begin{cases}\left|\Gamma_{i}^{-}\right| \widetilde{\rho}_{A_{i}}^{-} & \text {if } \mathbf{u}_{i}^{\star-} \cdot \mathbf{n}_{i}^{-} \leq 0, \\
\left|\Gamma_{i}^{-}\right| \widetilde{\rho}_{A}^{-} & \text {if } \mathbf{u}_{i}^{\star-} \cdot \mathbf{n}_{i}^{-}>0 .\end{cases}
\end{gathered}
$$

To be more specific, an order one approximation (with respect to space) is obtained by choosing $\widetilde{\rho}_{A}^{+}=\widetilde{\rho}_{A}^{-}=\rho_{A}$ and $\widetilde{\rho}_{A_{i}}^{+}=\widetilde{\rho}_{A_{i}}^{-}=\rho_{A_{i}}$. The second order accuracy in space can be reached by using a 
MUSCL technique [VL79, VL84] to increase the order of interpolation. In that case we define $C_{i}^{-}$ (respectively $C_{i}^{+}$) as the middle of $\Gamma_{i}^{-}$(respectively $\Gamma_{i}^{+}$), and we use :

$$
\begin{aligned}
& \widetilde{\rho}_{A}^{-}=\rho_{A}+\nabla^{-} \rho_{A} \cdot \mathbf{A} \mathbf{C}_{\mathbf{i}}^{-}, \quad \tilde{\rho}_{A}^{+}=\rho_{A}+\nabla^{+} \rho_{A} \cdot \mathbf{A C}_{\mathbf{i}}^{+}, \\
& \widetilde{\rho}_{A_{i}}^{-}=\rho_{A_{i}}+\nabla^{-} \rho_{A_{i}} \cdot \mathbf{A}_{\mathbf{i}} \mathbf{C}_{\mathbf{i}}^{-}, \tilde{\rho}_{A_{i}}^{+}=\rho_{A_{i}}+\nabla^{+} \rho_{A_{i}} \cdot \mathbf{A}_{\mathbf{i}} \mathbf{C}_{\mathbf{i}}^{+},
\end{aligned}
$$

where $\nabla^{-} \rho_{A}, \nabla^{+} \rho_{A}, \nabla^{-} \rho_{A_{i}}$ and $\nabla^{+} \rho_{A_{i}}$ are evaluated by an averaged value between an upstream and a downstream gradient, defined by the so-called $\beta$ scheme [DGS87] with the choice $\beta=1 / 3$. More precisely, the gradients $\nabla^{-} \rho_{A}$ and $\nabla^{+} \rho_{A}$ are defined as :

$$
\nabla^{-} \rho_{A}=\beta \overline{\nabla \rho_{A}}+(1-\beta) \underline{\nabla^{-} \rho_{A}}, \quad \nabla^{+} \rho_{A}=\beta \overline{\nabla \rho_{A}}+(1-\beta) \underline{\nabla^{+} \rho_{A}},
$$

with

$$
\frac{\sum_{j=1}^{n t}\left|M_{j}\right|(\nabla \rho)_{\left.\right|_{M_{j}}}}{\sum_{j=1}^{n t}\left|M_{j}\right|} \quad, \quad \underline{\nabla^{-} \rho_{A}}=\nabla \rho_{\left.\right|_{M_{i-1}}} \quad, \quad \underline{\nabla^{+} \rho_{A}}=\nabla \rho_{\left.\right|_{M_{i}}} .
$$

A similar procedure is used to derive $\nabla^{-} \rho_{A_{i}}$ and $\nabla^{+} \rho_{A_{i}}$. A flux limiter is finally added to ensure stable simulations and to avoid spurious oscillations in the vicinity of the discontinuities (see e.g. [BR96]).

Remark 1 Since we use an explicit method for evaluating the density, we are faced to the question of using time and space steps compatible to guaranty the stability of the scheme. This leads to a condition looking like $\Delta t=C h$, where $C$ is proportional to the inverse of the $L^{\infty}$ norm of the velocity.

\subsection{Compatibility Conditions}

\subsubsection{Velocities at the Interface of the Control Volume}

As explained in Section 2.3.2, the FV scheme needs at each time step of the simulation the determination of a auxiliary velocity $\mathbf{u}^{\star}$, defined on the interfaces of the control volume, from the knowledge of a function $\mathbf{u}$, defined on the domain $\Omega$. Our constraint is two-fold:

- First, we are concerned with a divergence free velocity field. Accordingly, as said in the Introduction, if we start from a homogeneous density, then, the scheme should produce a homogeneous solution.

- Second, the field $\mathbf{u}=(u, v)$ actually comes from a $\mathbb{P}_{2} \mathrm{FE}$ approximation. Therefore, it is a piecewise polynomial function which satisfies the divergence free constraint in the following weak sense

$$
\int_{\Omega} \operatorname{div}_{\mathbf{x}} \mathbf{u} \psi_{A} \mathrm{~d} \mathbf{x}=0
$$


where $\psi_{A}$ is the $\mathbb{P} 1$ basis function associated to the node $A$ (common to the triangles $M_{1}, \ldots, M_{n t}$ ). Since $\mathbf{u}$ is $\mathbb{P}_{2}$, we can compute the divergence by involving the values of the function at the points $A, A_{i}, A_{i}^{\prime}, A_{i, j}$ and $A_{i, j}^{\prime}$ : for $1 \leq i \leq n t$, we get (with the notation of Section 2.3.1)

$$
\begin{aligned}
6 \int_{M_{i}} \operatorname{div}_{\mathbf{x}} \mathbf{u} \psi_{A} \mathrm{~d} \mathbf{x}= & \left(y_{i+1}-y\right)\left(-u+u_{i}^{\prime}+u_{i, i+1}-u_{i+1}^{\prime}\right) \\
& +\left(y-y_{i}\right)\left(-u-u_{i}^{\prime}+u_{i, i+1}+u_{i+1}^{\prime}\right) \\
& +\left(x-x_{i+1}\right)\left(-v+v_{i}^{\prime}+v_{i, i+1}-v_{i+1}^{\prime}\right) \\
& +\left(x_{i}-x\right)\left(-v-v_{i}^{\prime}+v_{i, i+1}+v_{i+1}^{\prime}\right) .
\end{aligned}
$$

As a consequence, (2.23) becomes

$$
\begin{aligned}
6 \int_{\Omega} \operatorname{div}_{\mathbf{x}} \mathbf{u} \psi_{A} \mathrm{~d} \mathbf{x}= & \sum_{i=1}^{n t}\left(y_{i}-y\right)\left(u_{i-1}^{\prime}+u_{i-1, i}-u_{i, i+1}-u_{i+1}^{\prime}\right) \\
& +\sum_{i=1}^{n t}\left(x_{i}-x\right)\left(v_{i, i+1}+v_{i+1}^{\prime}-v_{i-1}^{\prime}-v_{i-1, i}\right)=0 .
\end{aligned}
$$

It is remarkable that the divergence-free constraint (2.25) does not involve the value of $\mathbf{u}$ at the vertices $A_{i}$ themselves $(1 \leq i \leq n t)$, but only in the middle of the edges. Now, let us define the auxiliary velocity $\mathbf{u}^{\star}$ : coming back to $(2.20)$ for a homogeneous state, the solution remains constant when $\mathbf{u}^{\star}$ fulfills the relation

$$
\sum_{i=1}^{n t}\left(\mathbf{u}_{i}^{\star-} \cdot \mathbf{n}_{i}^{-}\left|\Gamma_{i}^{-}\right|+\mathbf{u}_{i}^{\star+} \cdot \mathbf{n}_{i}^{+}\left|\Gamma_{i}^{+}\right|\right)=0 .
$$

The relation $(2.26)$ can be written as :

$$
\begin{array}{r}
\sum_{i=1}^{n t}\left(y_{i}-y\right)\left(\frac{u_{i-1}^{\star+}}{3}-\frac{u_{i}^{\star+}}{6}-\frac{u_{i+1}^{\star-}}{3}+\frac{u_{i}^{\star-}}{6}\right) \\
+\sum_{i=1}^{n t}\left(x_{i}-x\right)\left(-\frac{v_{i-1}^{\star+}}{3}+\frac{v_{i}^{\star+}}{6}+\frac{v_{i+1}^{\star-}}{3}-\frac{v_{i}^{\star-}}{6}\right)=0 .
\end{array}
$$

Therefore, identifying (2.25) with (2.27), we arrive at the following necessary and sufficient condition

$$
\mathbf{u}_{i-1}^{\prime}+\mathbf{u}_{i-1, i}-\mathbf{u}_{i, i+1}-\mathbf{u}_{i+1}^{\prime}=2 \mathbf{u}_{i-1}^{\star+}-\mathbf{u}_{i}^{\star+}-2 \mathbf{u}_{i+1}^{\star-}+\mathbf{u}_{i}^{\star-} .
$$

We search for $\mathbf{u}_{i}^{\star+}$ (respectively $\mathbf{u}_{i}^{\star-}$ ) as a convex combination of $\mathbf{u}_{i}^{\prime}, \mathbf{u}_{i, i+1}, \mathbf{u}_{i+1}^{\prime}$ (respectively $\mathbf{u}_{i}^{\prime}$, $\mathbf{u}_{i-1, i}, \mathbf{u}_{i-1}^{\prime}$ ) and we end up with the following definition of the auxiliary velocity

$$
\begin{aligned}
& \mathbf{u}_{i}^{\star+}=\frac{1}{3}\left(\mathbf{u}_{i}^{\prime}+\mathbf{u}_{i, i+1}+\mathbf{u}_{i+1}^{\prime}\right) \\
& \mathbf{u}_{i}^{\star-}=\frac{1}{3}\left(\mathbf{u}_{i-1}^{\prime}+\mathbf{u}_{i-1, i}+\mathbf{u}_{i}^{\prime}\right) .
\end{aligned}
$$


Finally, we also need to associate to the FV approximation of the density a piecewise polynomial approximation for the FE step. In our approach, the FV method uses a dual mesh and the control volumes, associated to the vertices of the triangles $K \in \mathcal{T}_{h}$, are formed from faces of the dual mesh connecting the barycenter of triangles through medians. To be more specific, if the FE is the classical Lagrange conforming linear element where degrees of freedom are associated to the vertices, for the control volume $\mathcal{C}_{i}$ corresponding to the $i$ - th degree of freedom, we identify :

$$
\sum_{i=1}^{M} \rho_{i} \chi_{\mathcal{C}_{i}}=\sum_{i=1}^{M} \rho_{i} \psi_{i}
$$

where $\chi_{\mathcal{C}_{i}}(\mathbf{x})=1$ if $\mathbf{x} \in \mathcal{C}_{i}$, and $\chi_{\mathcal{C}_{i}}(\mathbf{x})=0$ otherwise, $\psi_{i}$ is the $i-t h$ basis function of the FE space $Q_{h}$ and $M=\operatorname{dim}\left(Q_{h}\right)$.

\subsubsection{Some Remarks}

1. We remark that (2.29) and (2.30) imply $\mathbf{u}_{i}^{\star+}=\mathbf{u}_{i+1}^{\star-}$. However we point out that this velocity does not coincide with $\mathbf{u}\left(A_{i, i+1}^{\prime}\right)$ as it could be evaluated by using the $\mathbb{P} 2 \mathrm{FE}$ nature of $\mathbf{u}$ in the triangle. Actually, the formula for $\mathbf{u}_{i}^{\star+}=\mathbf{u}_{i+1}^{\star-}$ coincides with a linear 2D interpolation at $A_{i, i+1}^{\prime}$ from velocities known at the nodes $A_{i}^{\prime}, A_{i+1}^{\prime}$ and $A_{i, i+1}$.

2. In the case of a structured mesh (see Figure 2-(a)), the nodes $A_{i, i+1}^{\prime}, A_{i+1}^{\prime}$ and $A_{i+1, i+2}^{\prime}$ are on the same line $(1 \leq i \leq n t)$. Hence, it is tempting in this specific situation to define a global velocity $\mathbf{u}_{i}^{\star}$ constant on the segment $\Gamma_{i}=\Gamma_{i}^{-} \cup \Gamma_{i}^{+}$, by the formula

$$
\mathbf{u}_{i}^{\star}=\frac{\left|\Gamma_{i}^{+}\right| \mathbf{u}_{i}^{\star+}+\left|\Gamma_{i}^{-}\right| \mathbf{u}_{i}^{\star-}}{\left|\Gamma_{i}\right|} .
$$

Dealing with general unstructured meshes, it is also possible to define a auxiliary velocity $\mathbf{u}^{\star}$ which is constant on $\Gamma_{i}$, but the ponderation coefficients must be defined componentwise

$$
\begin{aligned}
& u_{i}^{\star}=u_{i}^{\star+}\left(\frac{y_{i+1}-y+\frac{y}{2}-\frac{y_{i}}{2}}{y_{i+1}-y_{i-1}}\right)+u_{i}^{\star-}\left(\frac{y-y_{i-1}-\frac{y}{2}+\frac{y_{i}}{2}}{y_{i+1}-y_{i-1}}\right) \\
& v_{i}^{\star}=v_{i}^{\star+}\left(\frac{x_{i+1}-x+\frac{x}{2}-\frac{x_{i}}{2}}{x_{i+1}-x_{i-1}}\right)+v_{i}^{\star-}\left(\frac{x-x_{i-1}-\frac{x}{2}+\frac{x_{i}}{2}}{x_{i+1}-x_{i-1}}\right)
\end{aligned}
$$

The geometrical interpretation of these relations is straightforward. For example on the mesh of the Figure 1, with $i=2$, we have : 


$$
\begin{aligned}
& u_{2}^{\star}=\frac{\alpha_{y}^{+}}{\beta_{y}} u_{2}^{\star+}+\frac{\alpha_{y}^{-}}{\beta_{y}} u_{2}^{\star-}, \\
& v_{2}^{\star}=\frac{\alpha_{x}^{+}}{\beta_{x}} v_{2}^{\star+}+\frac{\alpha_{x}^{-}}{\beta_{x}} v_{2}^{\star-},
\end{aligned}
$$

where $\alpha_{y}^{+}, \alpha_{y}^{-}, \beta_{y}, \alpha_{x}^{+}, \alpha_{x}^{-}$and $\beta_{x}$ are indicated on the figure. Anyway, our simulations on unstructured meshes use a variable velocity $\mathbf{u}^{\star}$ and the definition (2.29) and (2.30).

3. For a structured mesh, the control volume $\mathcal{C}_{A}$ can be defined in a different way, as the square $\left(A_{B R}, A_{T R}, A_{T L}, A_{B L}\right)$ (see Figure 2-(b)). In that case, $\mathbf{u}^{\star}$ can be chosen constant on each of the four parts of $\partial \mathcal{C}_{A}=\left[A_{B R} A_{T R}\right] \cup\left[A_{T R} A_{T L}\right] \cup\left[A_{T L} A_{B L}\right] \cup\left[A_{B L} A_{B R}\right]$. We have to define these velocities in order to satisfy the property

$$
u_{R}^{\star}+v_{T}^{\star}-u_{L}^{\star}-v_{B}^{\star}=0 .
$$

To derive $u_{R}^{\star}$, we first define the value $u_{M 1}^{\star}$ (resp. $u_{M 6}^{\star}$ ) as the first component of the velocity at the barycenter of the triangle $M 1$ (resp. M6) evaluated by $u_{M 1}^{\star}=\left(u_{R}+u_{R R}+u_{T R}\right) / 3$ (resp. $\left.u_{M 6}^{\star}=\left(u_{B}+u_{B R}+u_{R}\right) / 3\right)$. Then we get

$$
u_{R}^{\star}=\frac{1}{2}\left(u_{M 1}^{\star}+u_{M 6}^{\star}\right)
$$

$v_{T}^{\star}, u_{L}^{\star}$ and $v_{B}^{\star}$ are evaluated in a similar way, and this choice was proved to verify $(2.32)$ because of $(2.23)$.

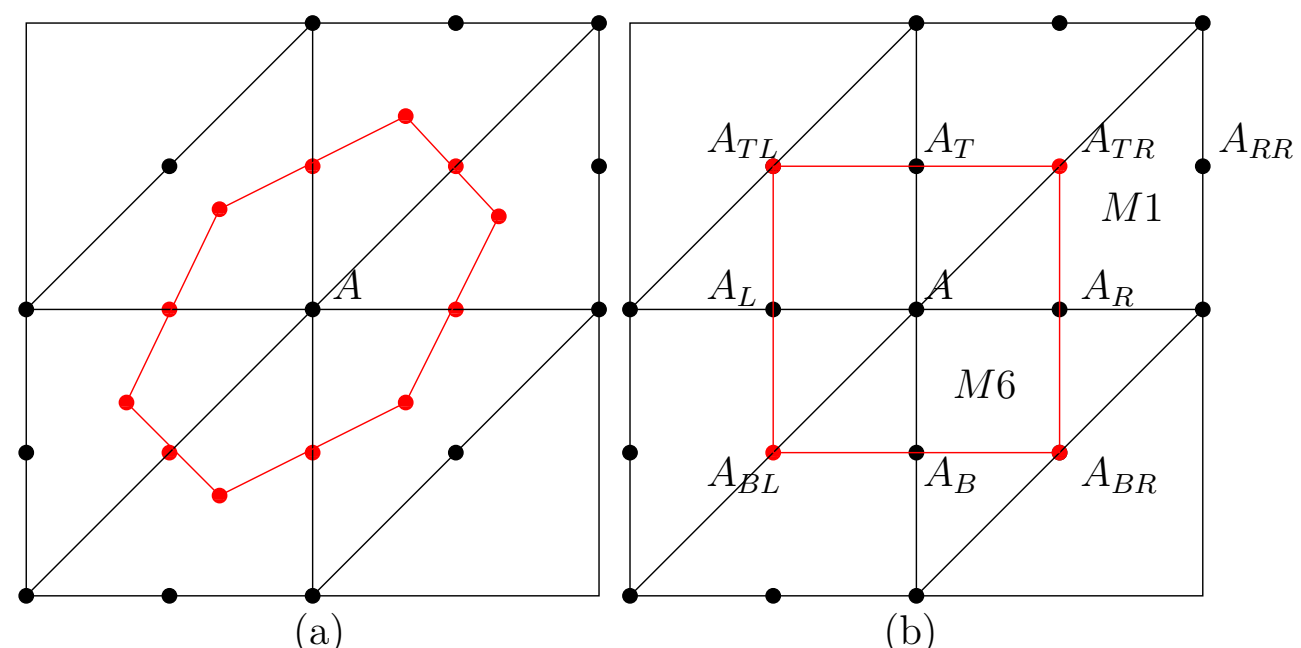

Figure 2: Control volume for $n t=6$ on structured mesh : The mesh defined in Remark 2: (a), and the mesh defined in Remark 3: (b). 


\subsubsection{Boundary Conditions}

A few words deserve to be said about the treatment of the boundary condition for the density by the FV scheme. Indeed, remind that the physical boundary condition for the mass conservation equation imposes the incoming flux $\rho \mathbf{u}_{\mid \Gamma_{\text {inc }}}=\rho_{\text {inc }}$ g. Then one needs to define a piecewise constant velocity on the interfaces of the control volumes belonging to the boundary of the computational domain $\partial \Omega_{h}$. Consider a vertex $A \in \partial \Omega_{h}$, belonging to $n t$ triangles (see Figure 3 for $n t=2$ ). We denote by $\mathbf{u}_{1}^{\star, \text { bd }}$ (respectively $\mathbf{u}_{n t+1}^{\star, \text { bd }}$ ) the velocity on the segment $\left[A_{1}^{\prime} A\right]$ (respectively $A A_{n t+1}^{\prime}$ ). We set $\mathbf{n}_{1}^{\text {bd }}$ (respectively $\mathbf{n}_{n t+1}^{\text {bd }}$ ) the unit outward normal vector on $\left[A_{1}^{\prime} A\right]$ (respectively $A A_{n t+1}^{\prime}$ ). We follow the same discussion as for interior volumes, bearing in mind to preserve the incompressibility condition.

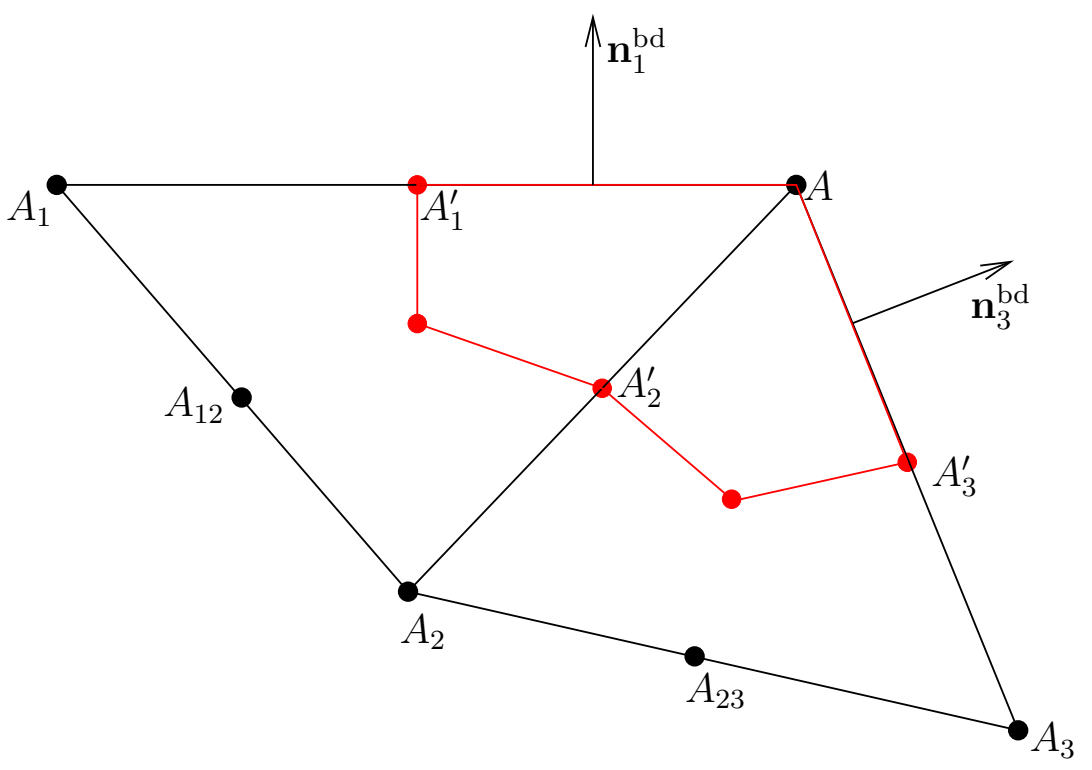

Figure 3: Boundary node $A$ on unstructured mesh for $n t=2$.

The question is to determine a velocity on the boundary $\partial \mathcal{C}_{A}$ of the control volume. For all interfaces belonging to the interior of $\Omega$, the velocity is derived as explained above; we are only left with the task of discussing the velocity to be imposed on $\partial \mathcal{C}_{A} \cap \partial \Omega_{h}$. Formula (2.24) still applies, but (2.25) is no more valid since now $A_{1}^{\prime} \neq A_{n t+1}^{\prime}$, with the convention used in subsection 2.3.1. We check that

$$
\int_{\partial \mathcal{C}_{A} \backslash \partial \Omega_{h}} \mathbf{u}^{\star} \cdot \mathbf{n} \mathrm{d} \gamma(\mathbf{x})-\int_{\Omega} \operatorname{div}_{\mathbf{x}} \mathbf{u} \psi_{A} \mathrm{~d} \mathbf{x}=-\left|\mathbf{A}_{\mathbf{1}}^{\prime} \mathbf{A}\right| \frac{2 \mathbf{u}_{1}^{\prime}+\mathbf{u}}{3} \cdot \mathbf{n}_{1}^{\mathrm{bd}}-\left|\mathbf{A}_{\mathbf{n t + 1}}^{\prime} \mathbf{A}\right| \frac{2 \mathbf{u}_{n t+1}^{\prime}+\mathbf{u}}{3} \cdot \mathbf{n}_{n t+1}^{\mathrm{bd}} .
$$


Therefore, to guaranty

$$
\int_{\partial \mathcal{C}_{A}} \mathbf{u}^{\star} \cdot \mathbf{n} \mathrm{d} \gamma(\mathbf{x})=0
$$

we are led to impose

$$
\mathbf{u}_{1}^{\star, \text { bd }}=\frac{2 \mathbf{u}_{1}^{\prime}+\mathbf{u}}{3}, \quad \mathbf{u}_{n t+1}^{\star, \mathrm{bd}}=\frac{2 \mathbf{u}_{n t+1}^{\prime}+\mathbf{u}}{3} .
$$

Finally, for the FV method, we distinguish two cases

- If $\mathbf{u}_{1}^{\star, b d} \cdot \mathbf{n}_{1}^{\text {bd }} \geq 0$, the flux is evaluated using the density as computed in the previous time step. According to the physical boundary condition, we do not need any further data in such a case of outgoing flux.

- If $\mathbf{u}_{1}^{\star, b d} \cdot \mathbf{n}_{1}^{\text {bd }}<0$, the flux is evaluated using the density provided by the data $\rho_{\text {inc }}$.

A similar construction is adopted for $n t+1$ replacing 1 .

\section{$3 \quad$ Numerical Results}

Let us now discuss the numerical results obtained with the hybrid FV-FE method described above. Let us recall that all simulations use the $\mathbb{P}_{2}-\mathbb{P}_{1} \mathrm{FE}$ approximation for computing the velocity and pressure fields, and the FV method detailed in the previous section for the density. It turns out that the second order scheme, including slope limiters if necessary, is perfectly appropriate.

\subsection{Validation of the Scheme}

First of all, we validate our numerical strategy. The first requirement is to recover results of usual simulations of the constant density case. Next, we check numerical rate of convergence by comparing to analytical solutions.

\subsubsection{Constant Density}

In Figure 4 we show the result of the computation for an initially homogeneous fluid for the standard lid-driven cavity test, at $R e=5000$. We indeed observe that the scheme preserves a constant density, and that the results for the velocity and the pressure perfectly coincide with computations that use a standard Navier-Stokes code. Therefore, our method does not introduce spurious variation of density. We also observe that this coupling does not introduce in this case a degradation of the accuracy.

\subsubsection{Analytical Solution, Rates of Convergence}

Next, we evaluate the abilities of the scheme to recover analytical solution and we check the rates of convergence. In particular, we shall make a couple of remarks concerning the structure of the 

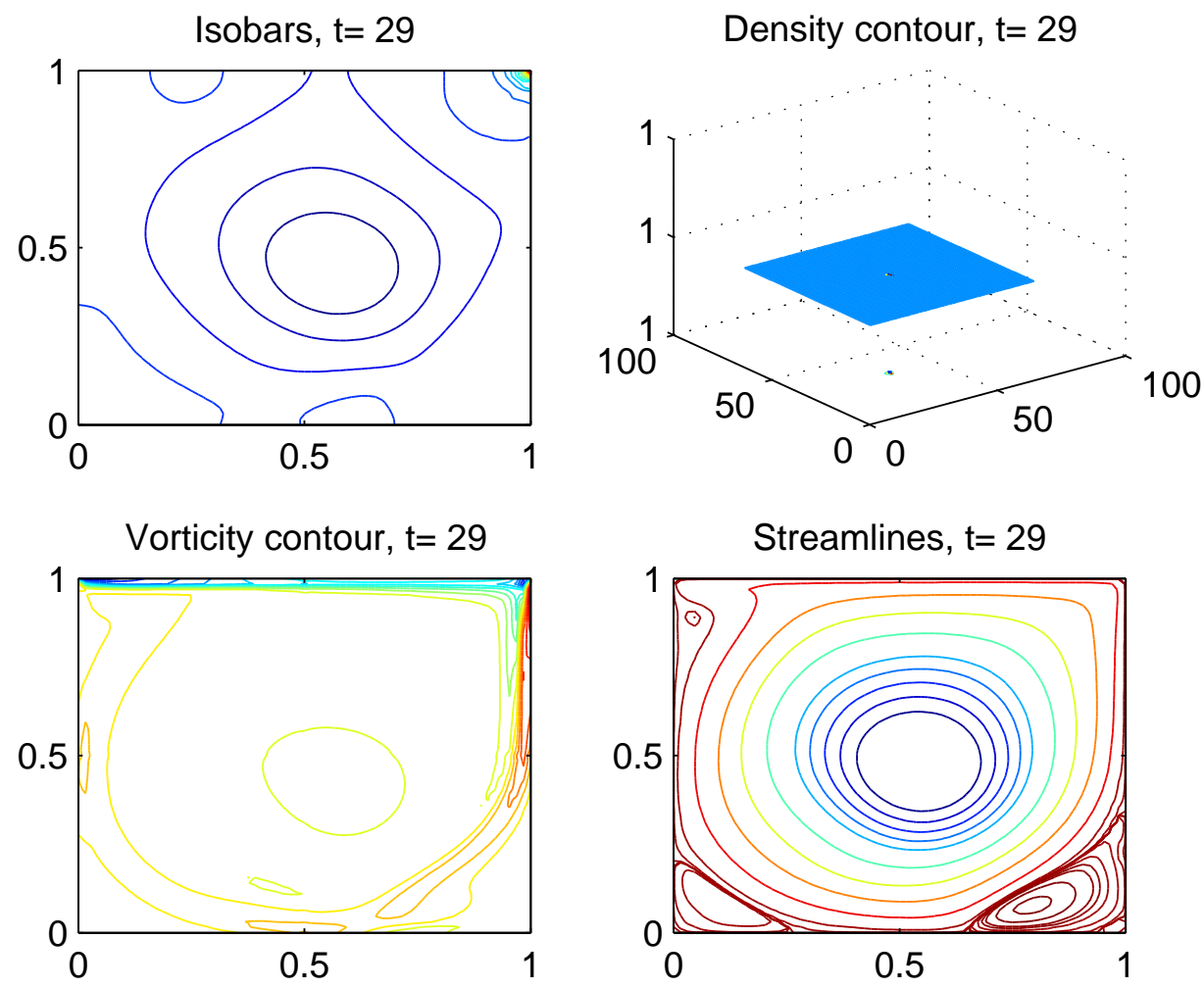

Figure 4: Velocity field, pressure and constant density for the lid-driven cavity test.

mesh that could be important for the performances of the scheme. We start with the example given in [GQ00] by :

$$
\left\{\begin{array}{l}
\rho_{\mathrm{ex}}(t, x, y)=\rho_{1}(r, \theta-\sin t), \\
\mathbf{u}_{\mathrm{ex}}(t, x, y)=\left(\begin{array}{l}
-y \cos t \\
x \cos t
\end{array}\right), \\
p_{\mathrm{ex}}(t, x, y)=\sin x \sin y \sin t,
\end{array}\right.
$$

where $\rho_{1}(r, \theta)=2+r \cos \theta$ and where $(r, \theta)$ are the usual polar coordinates. The fields $\rho_{\mathrm{ex}}(t, x, y)$ and $\mathbf{u}_{\mathrm{ex}}(t, x, y)$ satisfy the mass conservation equation (1.1) identically and $\mathbf{u}_{\mathrm{ex}}(t, x, y)$ is solenoidal. The momentum equation (1.2) is satisfied with the body force defined by

$$
\mathbf{f}_{\mathrm{ex}}(t, x, y)=\left(\begin{array}{c}
\left(y \sin t-x \cos ^{2} t\right) \rho_{\mathrm{ex}}(t, x, y)+\cos x \sin y \sin t \\
-\left(x \sin t+y \cos ^{2} t\right) \rho_{\mathrm{ex}}(t, x, y)+\sin x \cos y \sin t
\end{array}\right) .
$$

The computation has been performed for $0 \leq t \leq 0.5$ either on the square $[-1,1]^{2}$ or on the unit disk $0 \leq r \leq 1$. In the first case, we need to impose the incoming flux for the resolution of the mass conservation equation according to the discussion in Subsection 2.4.3. The convergence results are displayed by means of $h=h_{\max }$, where $h_{\max }$ is the length of the largest edge of the mesh. As explained above, the time step $\Delta t$ is proportional to $h$, see Remark 1 . For the velocity 
and the pressure, the errors are respectively evaluated using the usual $L^{2}(\Omega)$ norms $\left\|\mathbf{u}-\mathbf{u}_{h}\right\|_{L^{2}(\Omega)}$ and $\left\|p-p_{h}\right\|_{L^{2}(\Omega)}$. For the density, the error is evaluated with the usual $L^{1}(\Omega)$ norm, but using a reconstruction procedure similarly to [VM96]. Having at hand the piecewise constant density $\left(\rho_{h}\right)_{i}$ on each control volume $\mathcal{C}_{i}$ surrounding the node $A_{i}\left(x_{i}, y_{i}\right)$, we define on $\mathcal{C}_{i}$ a piecewise linear density reconstruction of $\left(\rho_{h}\right)_{i}$ by :

$$
\left(\tilde{\rho}_{h}\right)_{i}(x, y)=\left(\rho_{h}\right)_{i}+\left(\nabla \rho_{h}\right)_{i} \cdot\left(\begin{array}{c}
x-x_{i} \\
y-y i
\end{array}\right)
$$

where $\left(\nabla \rho_{h}\right)_{i}$ is a constant gradient on $\mathcal{C}_{i}$ evaluated by a weighted averaged computation from the gradients available on all the triangles surrounding $A_{i}$. The density error is then evaluated by $\left\|\rho-\tilde{\rho_{h}}\right\|_{L^{1}(\Omega)}$. The computations are performed in five different cases, to underline the influence of the mesh on the accuracy of the method :

a) The domain is the square which is discretized with a structured mesh, and the control volumes are defined as for an unstructured mesh (like in Figure 2 (a)). We obtain a covering of the computational domain by regular hexahedra;

b) The domain is the square which is discretized with the same structured mesh but where the control volumes are the squares (like in Figure 2 (b)); the method is described in Remark 3 in Section 2.4.2;

c) The domain is the square which is discretized with a general isotropic unstructured mesh (like in Figure 1);

d) The domain is the disk which is discretized with an isotropic unstructured mesh with some symmetric properties (Figure 5, left);

e) The domain is the disk which is discretized with a general isotropic unstructured mesh (Figure 5 , right) .

We plot in Figures 6 and 7 the error on the velocity, pressure and density for each of these cases. It shows the maximum error in time evaluated in $L^{2}(\Omega)$ (for velocity and pressure) or $L^{1}(\Omega)$ (for density) norm with respect to space. The lines corresponding to a rate of convergence of order one (slope 1) and order two (slope 2) are also displayed. The remarkable facts are the following:

- For the cases (a) and (b) (see Figure 6), we observe the optimal rates of convergence that can be expected from the method we use: namely order $\mathcal{O}\left(h^{2}\right)$ for all unknowns. Results obtained for the velocity and the pressure are nearly identical for this two cases. Note in particular that by construction of the scheme, the error on the velocity field is limited by the error made on the density. Since the interpolation error for the density is of order 2, we cannot expect more than order $\mathcal{O}\left(h^{2}\right)$ on the velocity, except when the density is a linear function of the space variable (in which case we can indeed obtain the $\mathcal{O}\left(h^{3}\right)$ error for the velocity at the price of using $\Delta t \simeq h^{3 / 2}$ ). 

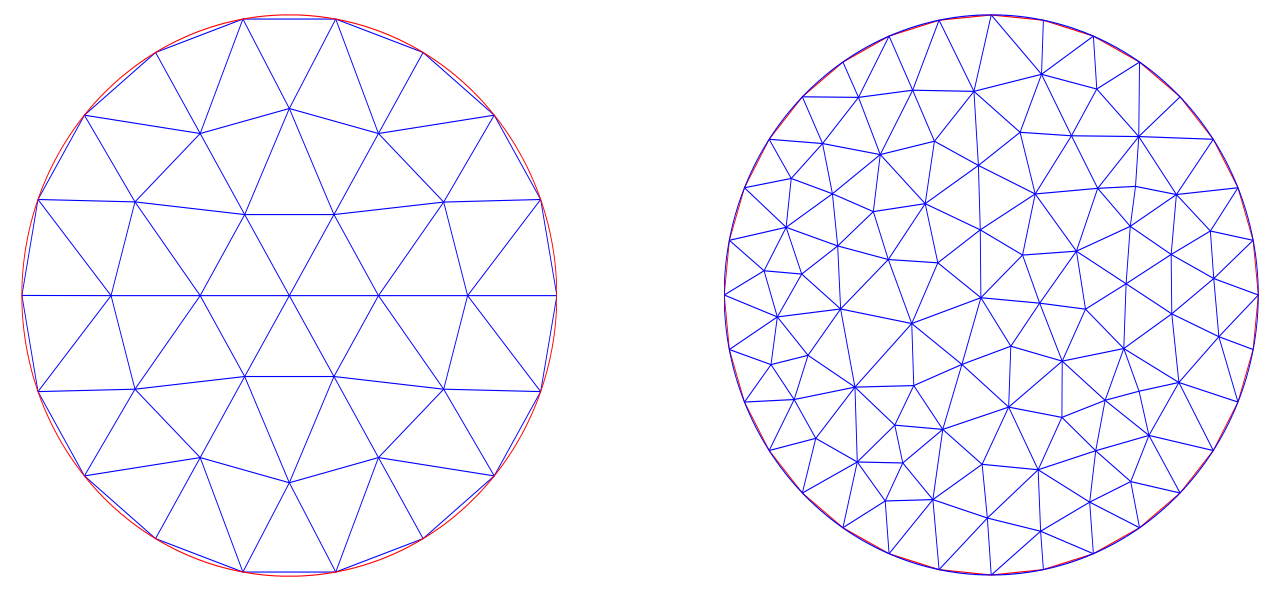

Figure 5: Unstructured meshes for the disk : symmetric one (left) and non symmetric one (right).
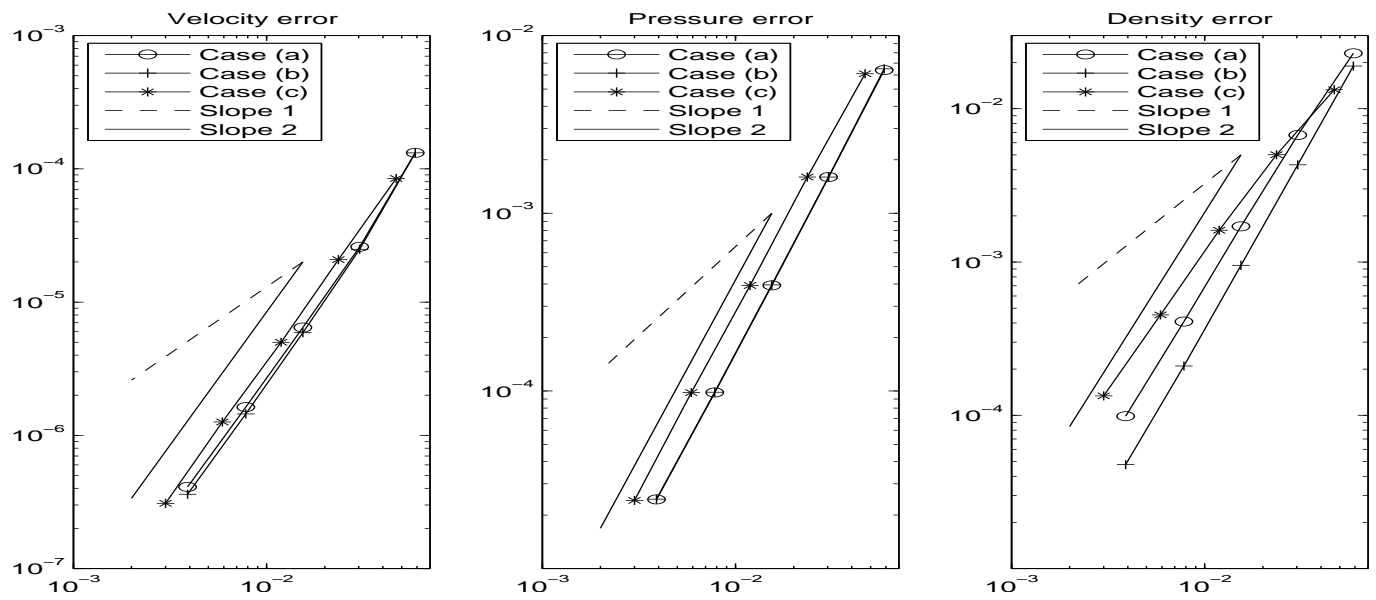

Figure 6: Rates of convergence for cases (a), (b) and (c). 

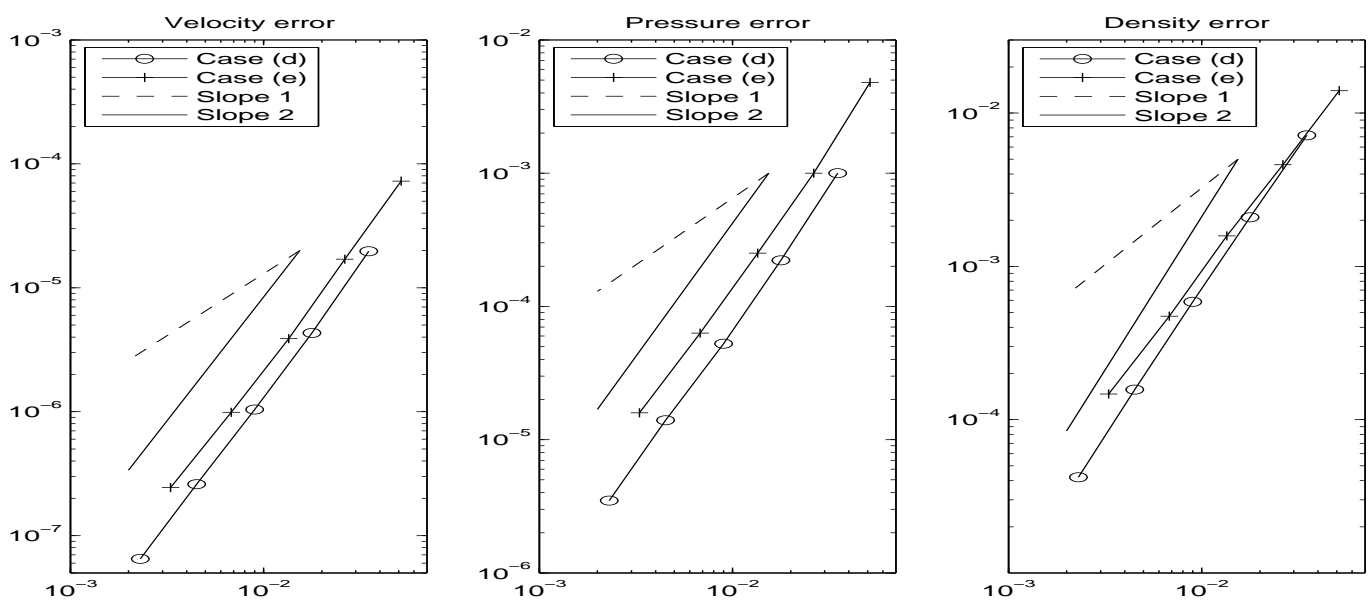

Figure 7: Rates of convergence for cases (d) and (e).

- For the case (c) (see Figure 6 again), the convergence rates remain in $\mathcal{O}\left(h^{2}\right)$ for the velocity and the pressure, and we observe a very slight degradation of the rates of convergence for the density (namely $\mathcal{O}\left(h^{1.80}\right)$ between the two most refined meshes). It is due to the unstructured topology of the mesh. Indeed, this phenomenon is typical of FV methods on complex meshes. A large bibliography is devoted to this topic and the order of convergence of FV methods on unstructured meshes, even for low-order methods and linear equations, is still an open problem [Des04a, Des04b, MV, MV07]. We also refer for studies on the effect of mesh geometries on the accuracy of FV methods to [BGP05] and the references therein. What is however remarkable is that despite this small loss of accuracy on the density, the accuracy on the velocity and the pressure remain optimal.

- For the cases (d) and (e) (see Figure 7), the convergence rates remain in $\mathcal{O}\left(h^{2}\right)$ for the velocity and the pressure, and we observe similarly to case (c) a slight loss of accuracy concerning the density, even if the order remains very satisfactory and definitely higher than one (namely $\mathcal{O}\left(h^{1.96}\right)$ for case $(\mathrm{d})$ and $\mathcal{O}\left(h^{1.62}\right)$ for case $\left.(\mathrm{e})\right)$. Once again, it is due to the unstructured topology of the mesh.

- Similar results are obtained by considering more complicated velocity or density fields, for instance having a polynomial dependence with higher degree with respect to the space variables, so that the exact solutions do not belong to the approximation spaces anymore.

In conclusion, we see that in the worst configuration (completely unstructured mesh on the disk), the rates of convergence are equal to $\mathcal{O}\left(h^{2}\right)$ for the velocity and the pressure and $\mathcal{O}\left(h^{1.62}\right)$ for the density. The proposed scheme is so proved to be very accurate. For the best one (structured mesh on the square), these rates are equal at least to $\mathcal{O}\left(h^{2}\right)$ for the three variables. This is the configuration chosen for the following numerical test (see subsection 3.2). 


\section{$3.2 \quad$ Viscous Rayleigh-Taylor Instability}

In this Section we deal with a physically more interesting problem, namely we investigate a RayleighTaylor instability. The problem has been considered in [BM92, GQ00, FGQ01] starting form the results and comments in [Try88] concerning the inviscid case. The fluid, initially at rest, is subject to gravity. It occupies the domain $(-d / 2, d / 2) \times(-2 d, 2 d)$, which splits into two region with varying density, the heavier fluid superposed to the light one. The interface is slightly smoothed since we set at time $t=0$ :

$$
\rho_{0}(x, y)=\frac{\rho_{\mathrm{m}}+\rho_{\mathrm{M}}}{2}+\frac{\rho_{\mathrm{M}}-\rho_{\mathrm{m}}}{2} \tanh \left(\frac{y-\eta \cos (2 \pi x / d)}{0.01 d}\right)
$$

with $\rho_{\mathrm{M}}>\rho_{\mathrm{m}}>0$, and $\eta>0$ the amplitude of the initial perturbation. The difficulty of the problem essentially depends on:

- the density ratio between the light and the heavy fluid, which is measured by the so-called Atwood number

$$
\mathrm{At}=\frac{\rho_{\mathrm{M}}-\rho_{\mathrm{m}}}{\rho_{\mathrm{M}}+\rho_{\mathrm{m}}}
$$

- the Reynolds number, defined as

$$
R e=\frac{\rho_{\mathrm{m}} d^{3 / 2} G^{1 / 2}}{\mu},
$$

where $\mu>0$ is the dynamic viscosity of the fluid (supposed to be constant in the whole domain) and $G$ is the gravitational acceleration.

The problem is very tough and it is difficult to distinguish the physical instabilities from instabilities having a purely numerical origin and which are amplified by the system. It turns out that the simulation are highly sensitive to

- the mesh orientation which can induce spurious drift velocity,

- the evaluation of the gravity term $\mathbf{f}=\rho \mathbf{g}$, where $\mathbf{g}=(0,-G)$,

- the treatment of the boundary condition.

Therefore, these points require a specific care to avoid spurious instabilities, which would definitely grow with time (actually exponentially fast, with a rate depending on the Reynolds number and the wavelength of the perturbation, according to the analysis in [Cha81]). Let us detail now the devices we use to remedy the difficulties.

- Mesh Orientation: Bearing in mind our results on the convergence rate of explicit solutions for structured and unstructured meshes, we give advantages to meshes having a lot of symmetries and in particular to structured meshes as defined in Figure 2-(b). Although the control volume is the square, uniform mesh orientations induce artificial drifts as shown in Figures 8 and 9. Indeed, these meshes produce small scale perturbations which grow with time, developing artificial drift-velocity near the vertical boundaries. These perturbations on the velocity field induce two second vortices which roll up on the upper part of the interface, following the orientation of the mesh (i.e. they spread in the domain in Figure 8 or live near the boundaries in Figure 9). To prevent this phenomena we use a triangular mesh with 
alternate directions, as shown in Figure 10. Of course, it would be possible to use also a fully unstructured mesh which is intended to average these mesh instabilities; it would also allow the use of mesh refinement strategies. We shall go back to this question elsewhere. Finally, it seems that structured meshes with alternate directions and with control volumes having a lot of symmetries but without being a simple square, are slightly more sensitive to develop instabilities. This certainly gives also advantages to methods based on very regular meshes like finite difference approaches [BM92, JL04] which, however, do not extend easily to complex geometries.
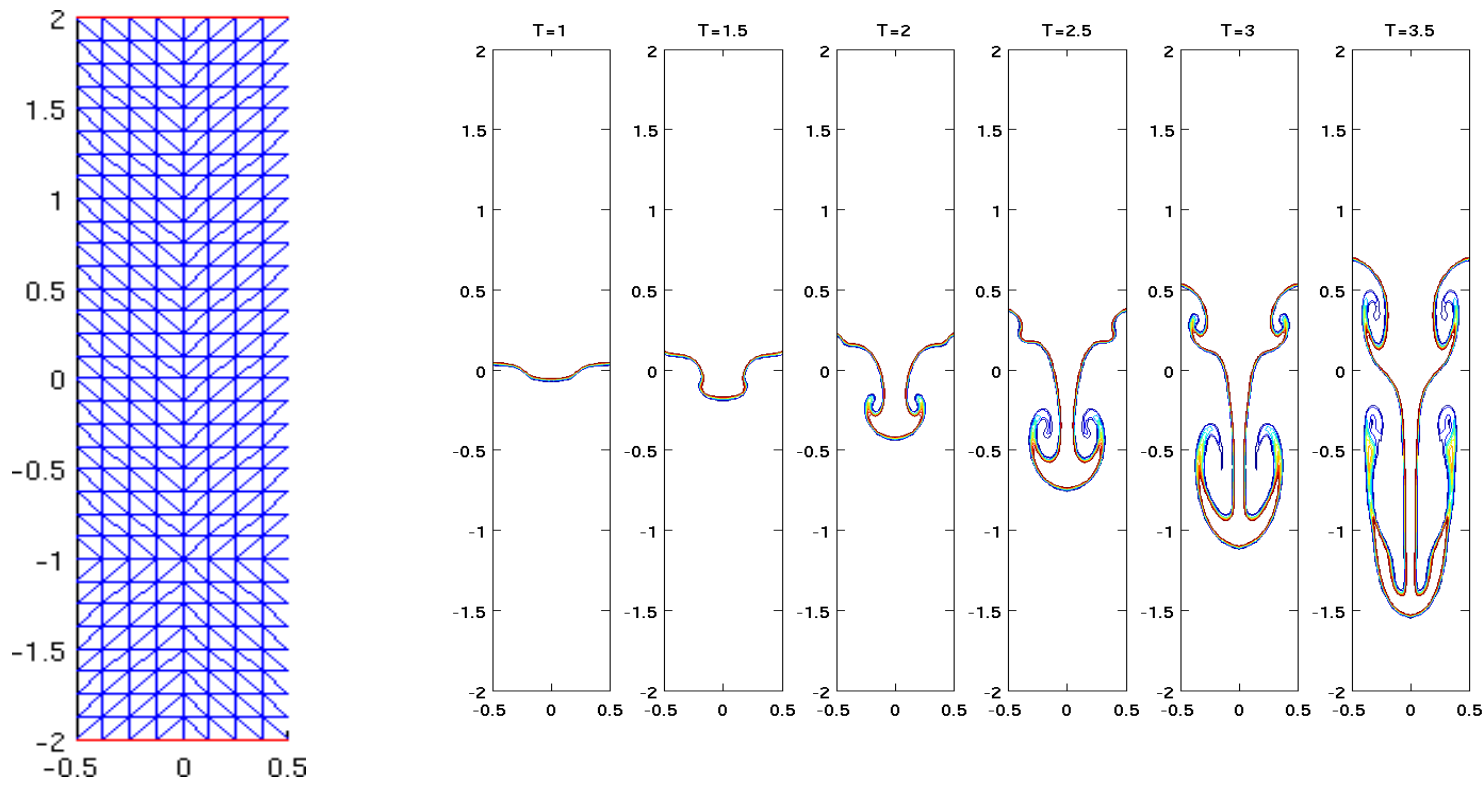

Figure 8: Rayleigh-Taylor instability: Instabilities due to mesh orientation, Example 1.

- Gravity Term: Since we have at hand 3 values of density per element when computing the velocity field, it would be quite natural to use a $\mathbb{P} 1$ interpolation of the density in the elements. This is the definition we adopt for the left hand side term of the momentum equation. However, for the evaluation of the gravity force, this choice can be improved having in mind the stable case of two fluids of different densities superposed one over the other with a flat interface. Indeed, for structured meshes like in Figures 8, 9 and 10, the interpolation introduces an initial sawtooth profile of the density, which then induces unphysical vertical velocities. Of course, this phenomenon is reduced as the mesh size tends to 0, but this simple example discriminates the $\mathbb{P} 1$ interpolation for evaluating the gravity term when dealing with unstable cases. Considering the same example, also a $\mathbb{P} 0$ interpolation of the density, obtained by the average of the three available values on an element, introduces a sawtooth profile of the density. However, other $\mathbb{P} 0$ interpolations of the density can be defined in order to produce a flat interface of the density. Among many different possible strategies, we define the gravity 

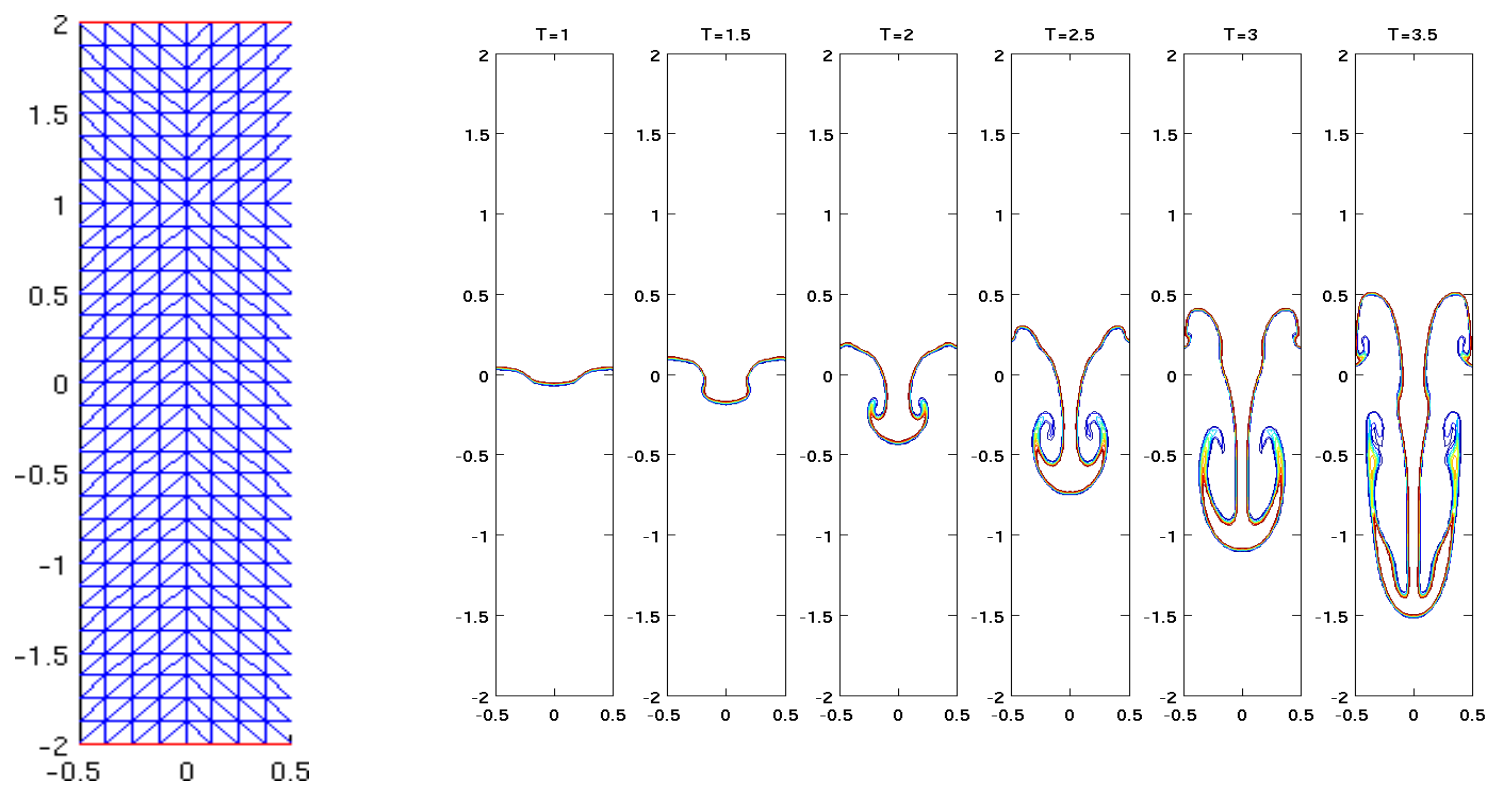

Figure 9: Rayleigh-Taylor instability: Instabilities due to mesh orientation, Example 2.
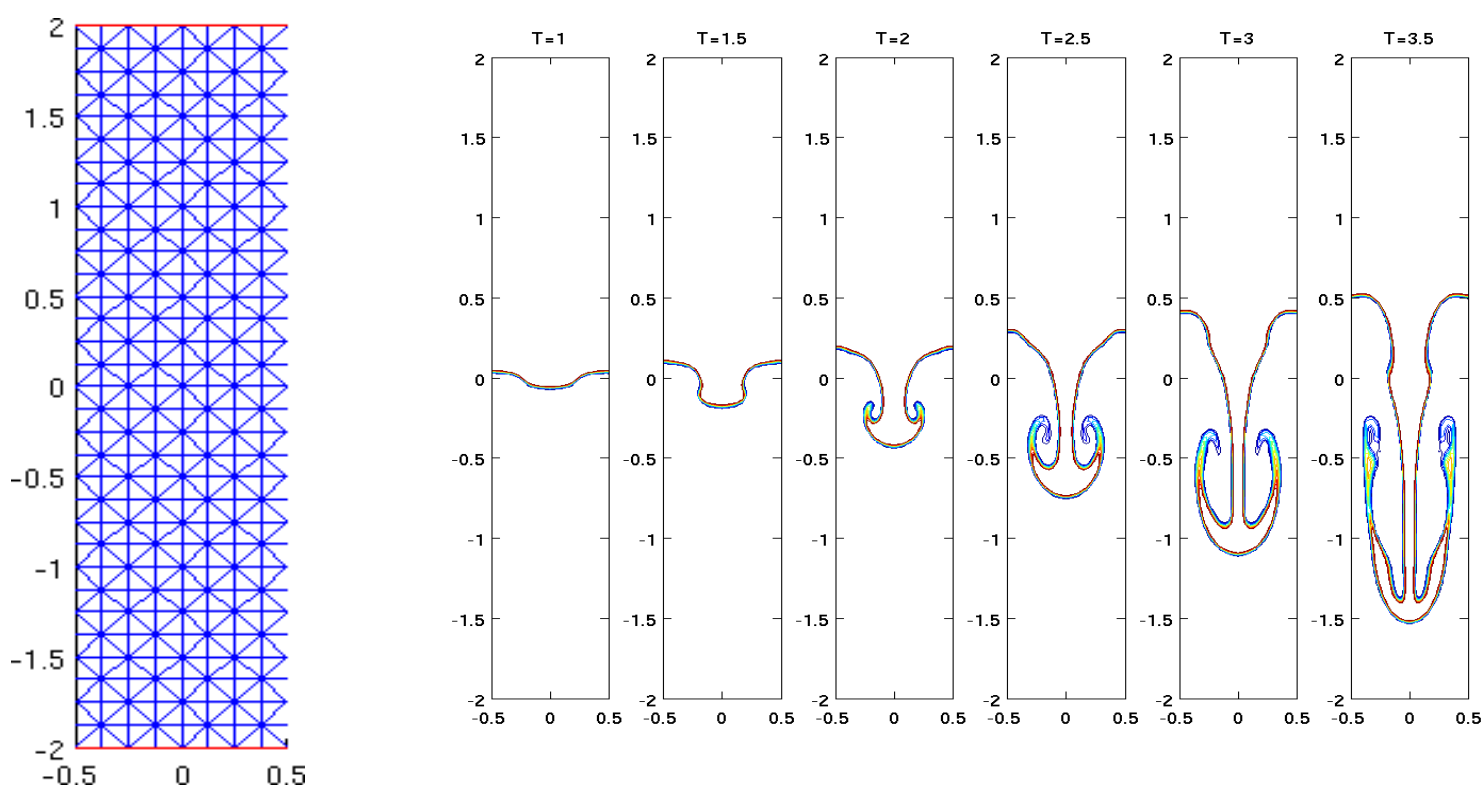

Figure 10: Rayleigh-Taylor instability: A performing uniform mesh. 
term in a given element by the constant value

$$
\frac{\rho_{-}+\rho_{+}}{2} G
$$

where $\rho_{-}$and $\rho_{+}$are the extreme values of the density present in the element. We could also use $\rho_{-}$or $\rho_{+}$as the constant value on an element, but this seems to be less stable, in particular as the Atwood number increases, with similar phenomena observed near the vertical boundaries in Figures 8 and 9 .

- Boundary Conditions: According to [Try88, BM92], the continuous problem is supplemented by no-slip boundary condition on the horizontal boundaries and periodicity conditions on vertical boundaries. Actually, according to [GQ00, FGQ01] and to numerical simulations presented in Figure 10, the solution has symmetries and we compute the solution on the half domain $(0, d / 2) \times(-2 d, 2 d)$ with the following boundary conditions for the velocity field: denoting $\mathbf{u}=(u, v)$, we impose

$$
\left\{\begin{array}{lll}
\text { On the horizontal boundaries: } & u=0, & v=0, \\
\text { On the vertical boundaries: } & u=0, & \partial_{x} v=0 .
\end{array}\right.
$$

For the density, we note that $\mathbf{u} \cdot \mathbf{n}$ always vanishes on the boundary so that there is no incoming flux. Then, we use the treatment described above in Section 2.4.3.

In what follows, the time variable is scaled as $t=t^{\prime} \sqrt{d / \operatorname{At} G}$, with $t^{\prime}$ dimensionless, according to [Try88]. We compare the solutions obtained at different Atwood numbers and for many Reynolds numbers.

- A low Atwood number problem : Setting At $=0.5\left(\rho_{\mathrm{M}}=3, \rho_{\mathrm{m}}=1\right)$, we begin with a low Reynolds case, $R e=1000$. For the initial condition we set $\eta=0.1$. The results displayed in Figure 11 are qualitatively close to those in [GQ00, FGQ01]. In this case, the calculation is quite indifferent to the choice of mesh orientation and to the evaluation of the gravity term. We observe that when the Reynolds number increases (see also Figures 12 for $R e=5000$ and 13 for $R e=20000$ ), the velocity of the characteristic mushroom shape is the same for all the Reynolds numbers considered. These qualitative results are in accordance with the analysis in [Cha81], for which the viscosity plays no role among the interface perturbations with very long wavelengths. The influence of the increasing Reynolds number appears in the shape of the rising counter-rotating vortices, which induces many different small structures for $t \geq 2$. It is of course difficult to assess the accuracy at large times and care must be taken that the resolution is sufficiently high. Again, some spurious instabilities seem to start near the right hand boundary for $R e=20000$ at $t=2$ and obviously they grow with time. A finer uniform mesh would cure this numerical phenomenon. Finally, comparing our results at $R e=5000$ with those presented in [GQ00, FGQ01], there is a satisfactory agreement of the global characteristics of the flow in the early stage and we can observe some slight discrepancies only at large times of the calculation. 

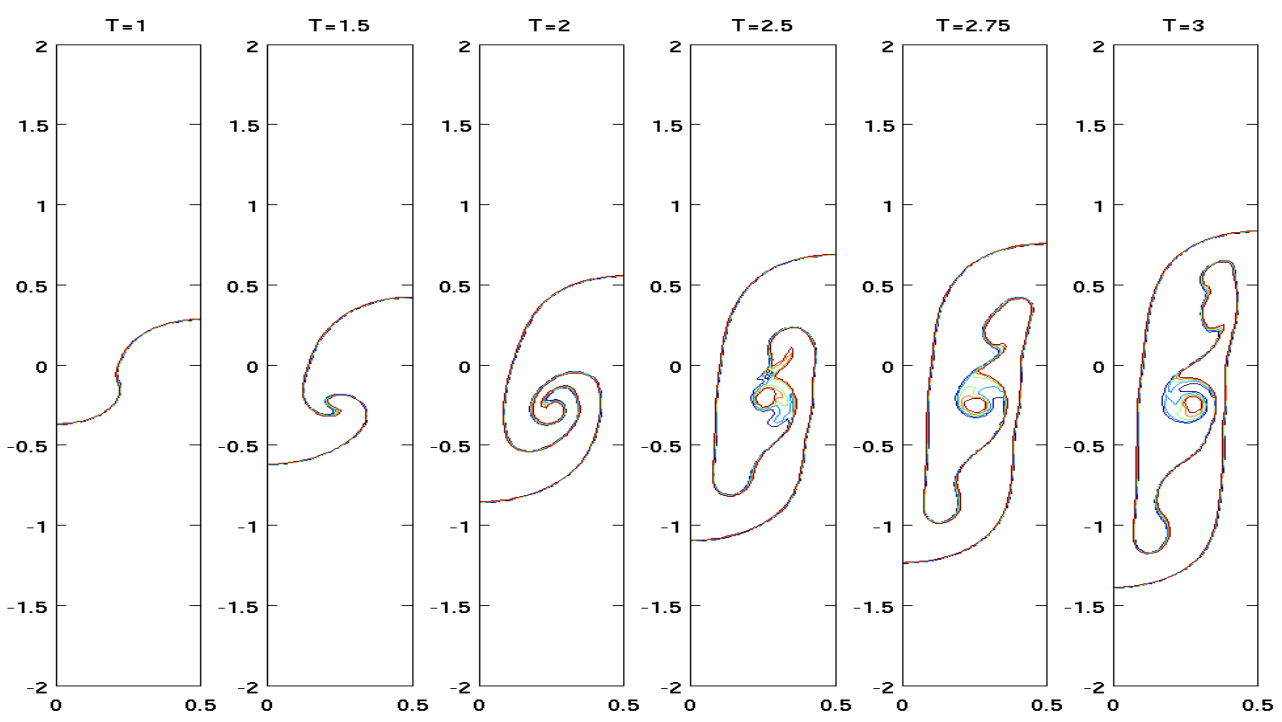

Figure 11: Rayleigh-Taylor instability: evolution of the interface; $R e=1000$, density ratio $=3$, initial amplitude $\eta=0.1$. Uniform Mesh $30 \times 240$, density contours $1.4 \leq \rho \leq 1.6$.
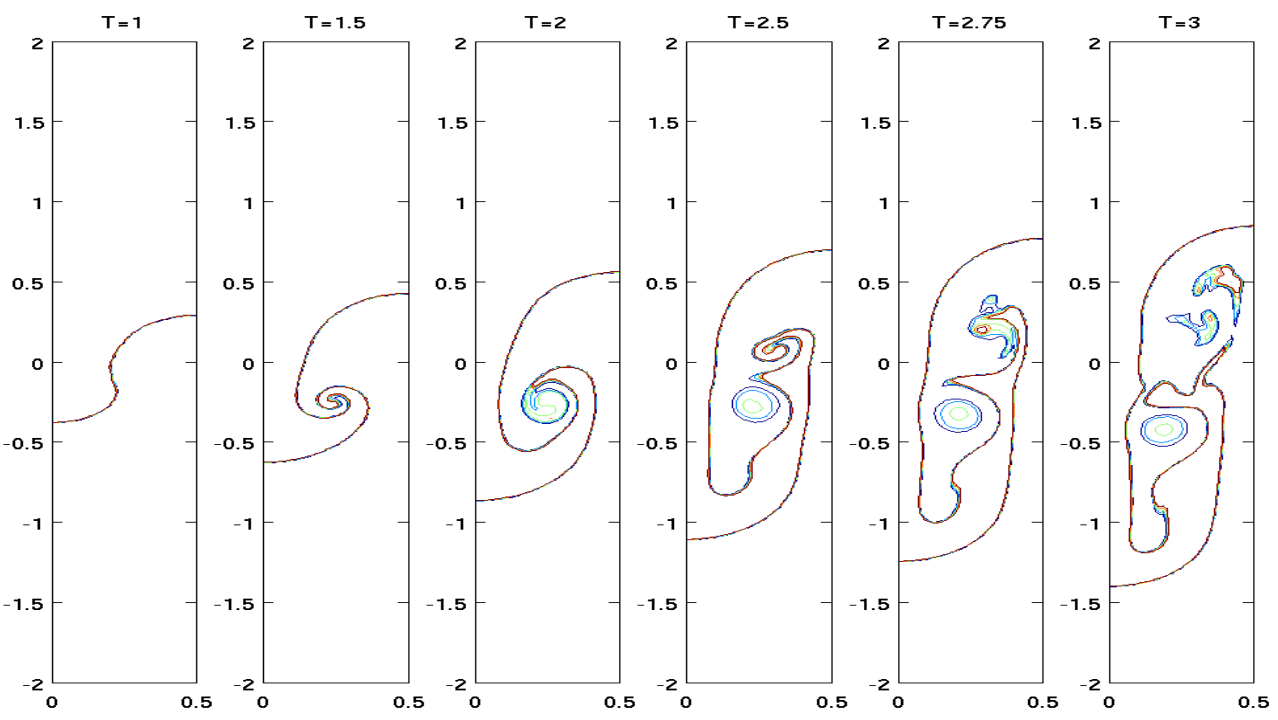

Figure 12: Rayleigh-Taylor instability: evolution of the interface; $R e=5000$, density ratio $=3$, initial amplitude $\eta=0.1$. Uniform Mesh $40 \times 320$, density contours $1.4 \leq \rho \leq 1.6$. 

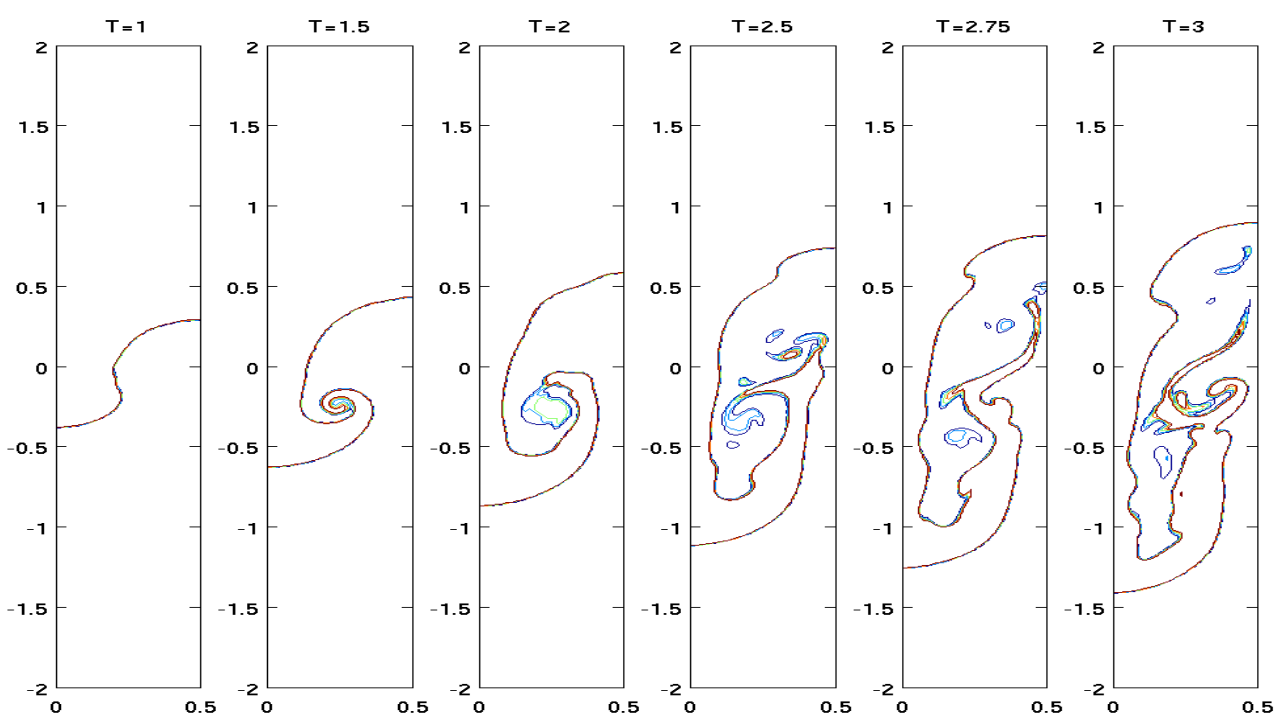

Figure 13: Rayleigh-Taylor instability: evolution of the interface; $R e=20000$, density ratio $=3$, initial amplitude $\eta=0.1$. Uniform Mesh $40 \times 320$, density contours $1.4 \leq \rho \leq 1.6$.

- A high Atwood number problem : Setting At $=0.75\left(\rho_{\mathrm{M}}=7, \rho_{\mathrm{m}}=1\right)$, we consider again two Reynolds numbers, $R e=1000$ and $R e=5000$. For the initial condition, let $\eta=0.01$ be the amplitude of the interface perturbation, like in [BM92, GQ00]. For $R e=1000$, the results displayed in Figure 14 can be compared with those of [GQ00] and [BM92]. In this case, more important discrepancies with [GQ00] appear. These discrepancies are first located at the right hand boundary and they are spread in the domain as time increases. Note that the behavior of the solution is far more sensitive to the numerical choices that for the low Atwood number, but our calculations remain qualitatively close to those in [BM92], especially in the early stage. As said above, it is difficult to assess the accuracy of the simulations at large times, and the comparison could become irrelevant since our space grid of resolution is much larger than that one used in [BM92]. At this high Atwood number, the roll up of the heavy fluid is less pronounced, compared to those obtained at low Atwood number (see Figures 11 and 14). Take care not to compare the evolution of the interface at the same nondimensional time, because the amplitude of the initial condition is not the same. As the heavy fluid continues to fall, the shape of the rolled-up vortex developed in the interior of the head presents less rich structures with respect to those displayed in [BM92]. Finally, comparing the evolution of the interface for $R e=1000$ and $R e=50000$ (see Figures 14 and 15), we can confirm that the viscosity plays no role on the velocity of downward motion of the heavy fluid. Slight discrepancies can be observed on the evolution of the upward motion of the light fluid and some spurious instabilities appear near the right hand at $t=2.5$ and obviously grow with time. Again, a finer uniform mesh would cure this numerical phenomenon.

- A very high Atwood number problem : Setting At $=0.9\left(\rho_{\mathrm{M}}=19, \rho_{\mathrm{m}}=1\right)$, we consider 

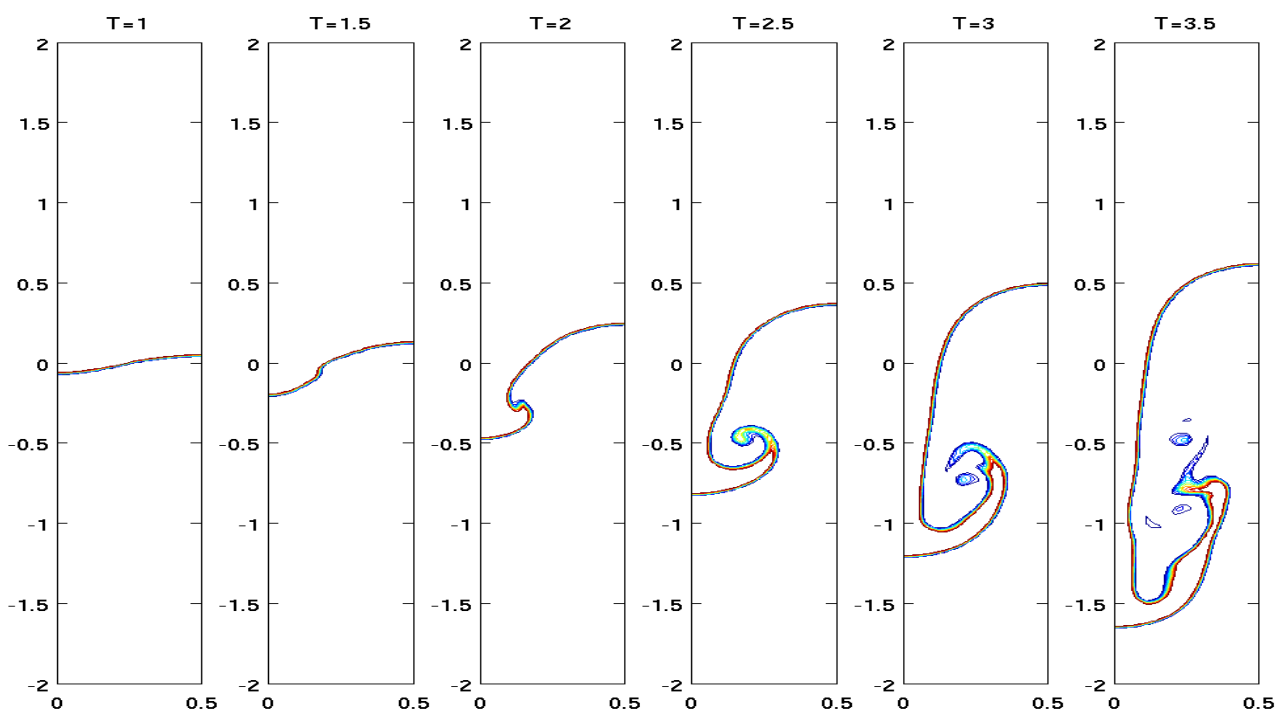

Figure 14: Rayleigh-Taylor instability: evolution of the interface; $R e=1000$, density ratio $=7$, initial amplitude $\eta=0.01$. Uniform Mesh $40 \times 320$, density contours $2 \leq \rho \leq 4$.
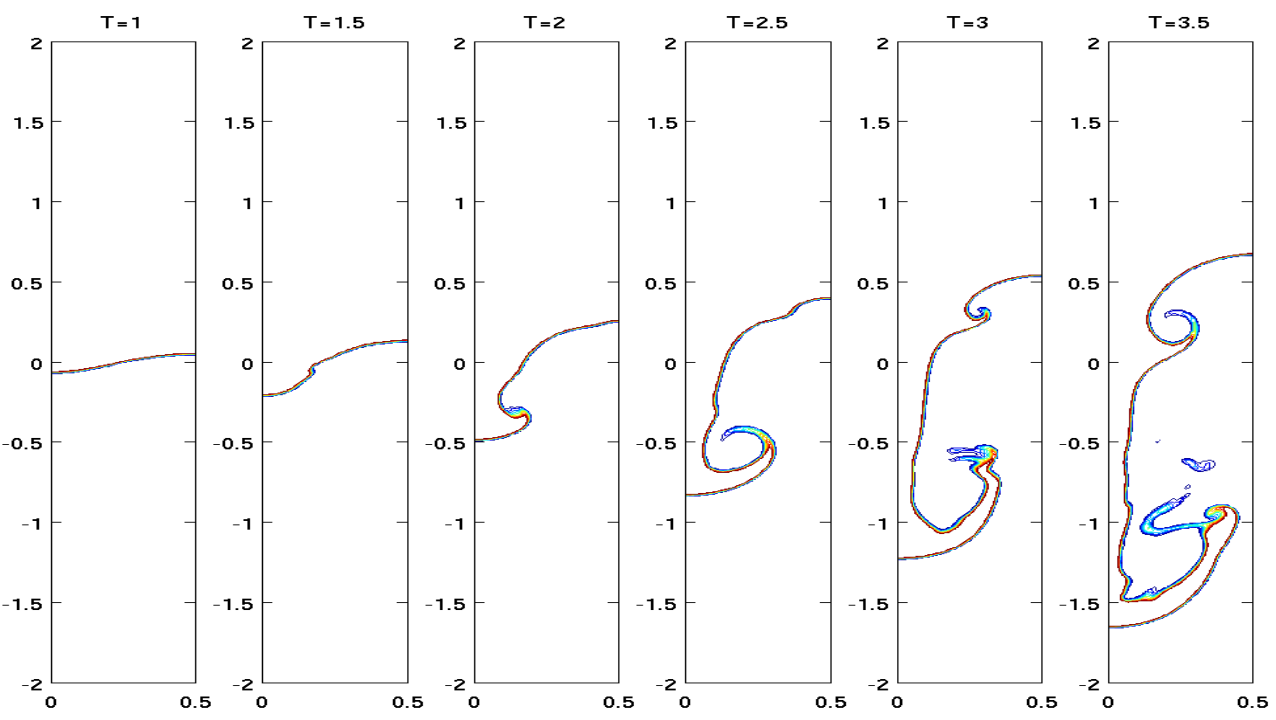

Figure 15: Rayleigh-Taylor instability: evolution of the interface; $R e=5000$, density ratio $=7$, initial amplitude $\eta=0.01$. Uniform Mesh $50 \times 400$, density contours $2 \leq \rho \leq 4$. 
only a low Reynolds case, $R e=1000$ (see Figure 16). As the Atwood value increases, the sensitiveness of the calculation to the numerical instabilities grows. The downward motion of the heavy fluid increases with the density difference and the solution develops the characteristic mushroom shape later than for the case At $=0.75$, without any structure in the interior of the head. These phenomena are observed comparing Figures 14 and 16, obtained with the same mesh and using the same amplitude $\eta=0.01$ of the initial interface perturbation. Moreover this Atwood dependent behavior was already observed in [Try88]. Notice that at At $=0.9$ it is very difficult to continue the simulation up to the nondimensional time $t=3$, and it seems that the evolution of the interface configuration does not change significantly when using finer grids. The application of a surface tension model may produce better results on the form of the spike and of the mushroom head at the tip of the spike. We shall go back to this question elsewhere.
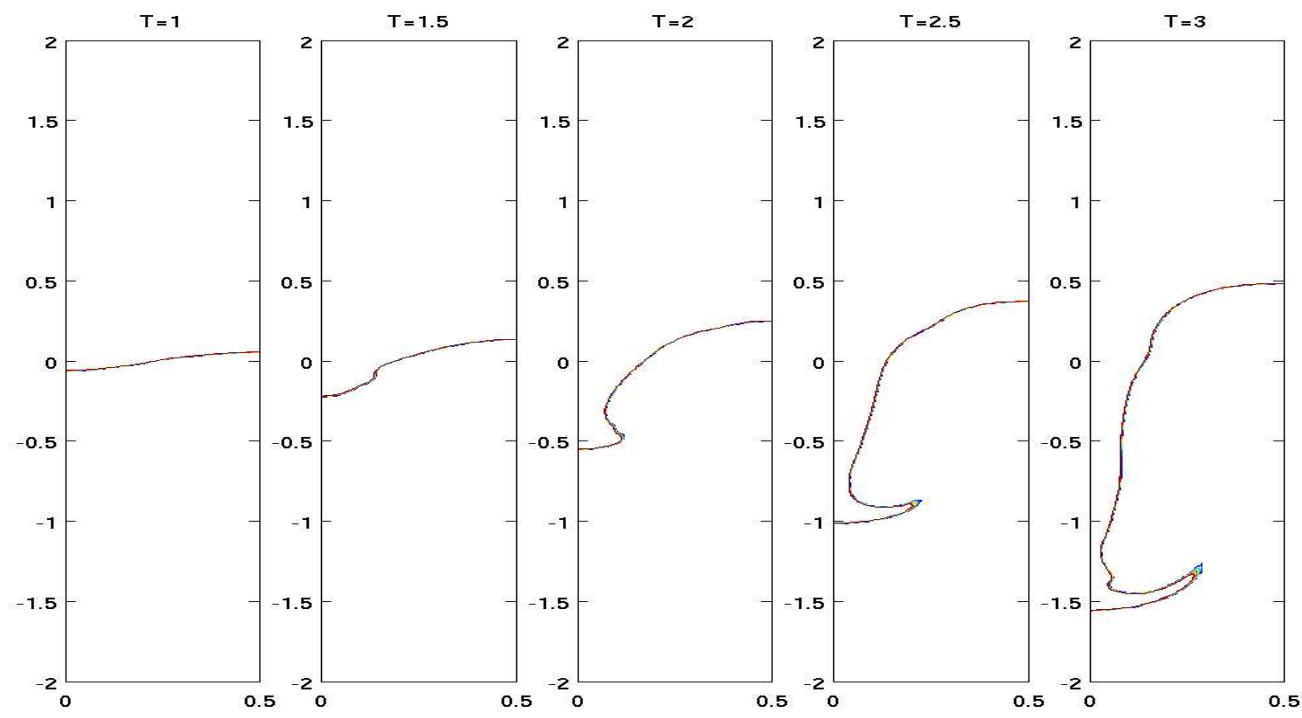

Figure 16: Rayleigh-Taylor instability: evolution of the interface; $R e=1000$, density ratio $=19$, initial amplitude $\eta=0.01$. Uniform Mesh $40 \times 320$, density contours $9 \leq \rho \leq 11$.

\subsection{Falling Bubble Test}

The goal of this test is to investigate the capability of our hybrid method to work with more large density variations. This simulation is inspired from [SBJK99], even if this paper is concerned with the Euler system. A heavy "droplet" falls through a light fluid and impacts into the plane surface of the heavy fluid in a cavity. The computational domain is $(0, d) \times(0,2 d)$, where $d=1$ and at $t=0$ the fluid is at rest with density:

$$
\rho(x, y)= \begin{cases}100 & \text { if } 0 \leq y \leq 1 \quad \text { or } 0 \leq r \leq 0.2 \\ 1 & \text { if } 1<y \leq 2 \text { or } 0.2<r\end{cases}
$$


where $r=\sqrt{(x-0.5)^{2}+(y-1.75)^{2}}$. As in [SBJK99], the equations are made dimensionless by using the following references: $\rho_{m}$ for density, $d$ for length, $\sqrt{d / G}$ for time, so that the reference velocity is $\sqrt{d G}$. By non-dimensionalization, the gravity term is $\mathbf{f}=(0,-\rho)$ and the Reynolds number is defined as in Section 3.2. In our test, the viscosity of the fluid is supposed to be constant in the whole domain and we have $R e=3132$. No surface tension model is taken into account, then a deformation of the spherical droplet can be observed. In this case, the difficulty comes from the discontinuous interface and the problem becomes harder as the difference between the densities increases (note that in the test of Rayleigh-Taylor instability, the interface was artificially smoothed). Figure 17 shows the evolution of density contours $\rho=[20,35,40,45,50,55,60,65,80]$, which are qualitatively close to those in [SBJK99], obtained with a larger density ratio. After the splash of the droplet, some areas of lighter density appear within the heavy fluid (in Figure 17, this corresponds to the iso-contours $\rho \geq 65$ ). This is consistent with the results shown in [SBJK99] and reference therein, and seems to correspond to a phenomenon for which some particles of lighter fluid are trapped in the heavy fluid after the splash. Finally, it is even difficult to conserve the equilibrium solution since vertical velocities are produced. Of course, the amplitude of these perturbations decreases with the size of the mesh, but it increases with the Reynolds number and the gradient of density. There is clearly a motivation here for developing mesh refinement strategies.

\section{Conclusion}

In this work, a new Finite Volume-Finite Element scheme was derived for the resolution of the incompressible Navier-Stokes system with variable density on unstructured meshes. The originality of our approach is, thanks to a time splitting procedure, to allow the resolution of the mass equation by a Finite Volume method and the resolution of the momentum equation associated to the divergence constraint by a Finite Element one. A fundamental point lies on the compatibility relation between the Finite Volume velocity field, which has to be defined on the interfaces of the control volumes, and the Finite Element velocity field which is a continuous elementwise polynomial function. Indeed, the divergence free constraint has to be preserved on both fields. Several other points were carefully described, like the choice of the control volumes in the particular case of the structured mesh or the boundary conditions treatment for the density, in order to provide a description as complete as possible. Four numerical tests underlined the capability of the scheme to ensure relevant simulations. First, the standard lid-driven cavity test with constant density was performed. Then, the rates of convergence of the method were proved to be in accordance with the theoretical expected ones, leading so to an accurate solver. The simulation of the viscous Rayleigh-Taylor instability was also investigated, and a meticulous study of some numerical choices was carried on (in particular concerning the mesh orientation and the gravity term). We obtained very good results, even for rather high Reynolds and Atwood numbers compared to the moderately refined meshes used. This robustness property of the scheme with regards to high density ratios was finally consolidated by the falling bubble test included a completely discontinuous interface. In a forthcoming paper, this hybrid scheme will be used for the simulation of a low Mach number model for which the divergence free condition on the velocity will be replaced by a relation between the divergence of the velocity and non linear derivatives of the density, together with the use of an 

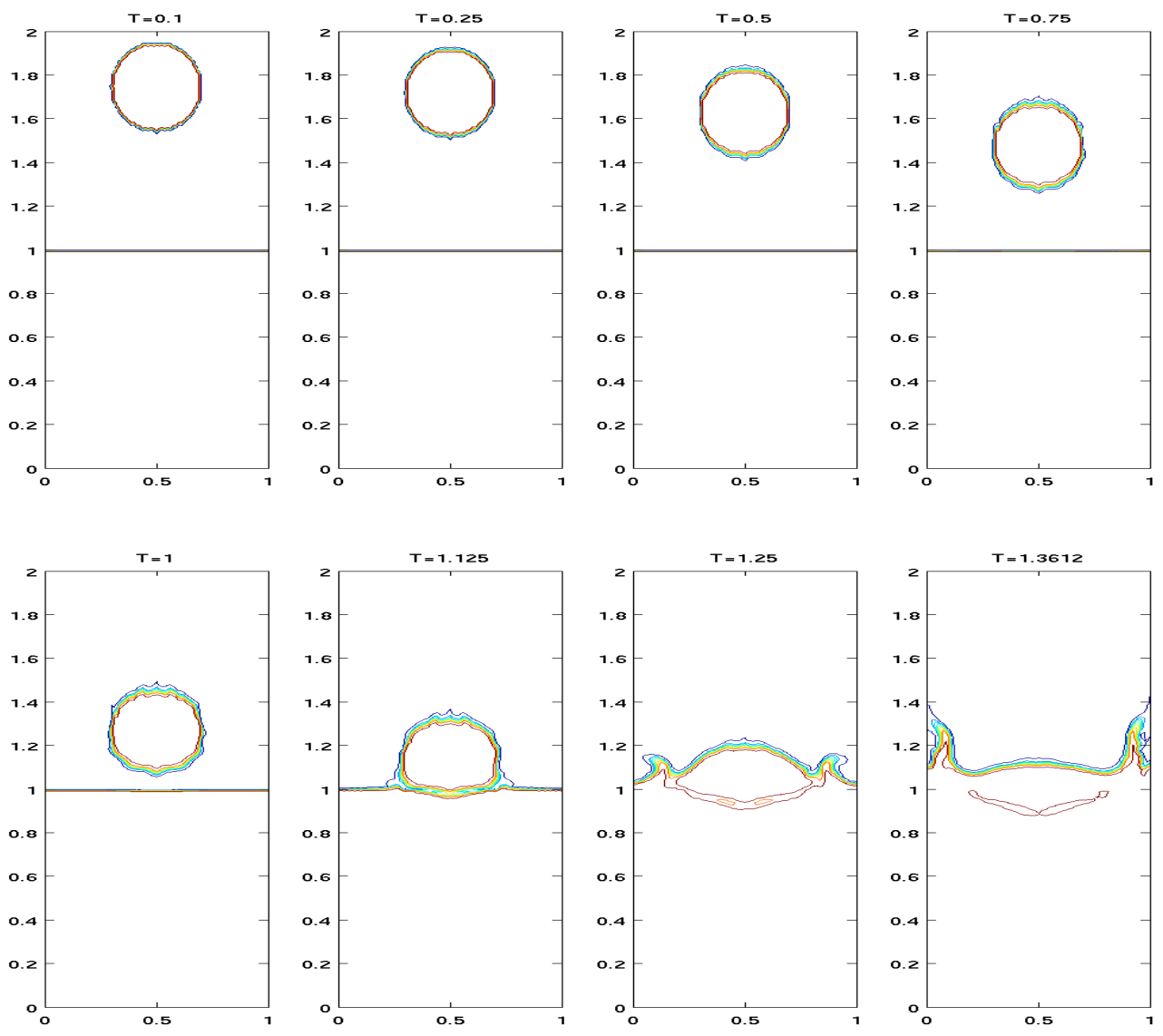

Figure 17: Falling bubble test: evolution of the interface; $R e=3132$, density ratio $=100$. Uniform Mesh $80 \times 160$, density contours $\rho=[20,35,40,45,50,55,60,65,80]$. 
adaptive mesh refinement strategy.

\section{Acknowledgment}

The authors would like to thank Frédéric Lagoutière for several very fruitful discussions about this work.

\section{References}

$\left[\mathrm{ABC}^{+} 98\right]$ A. S. Almgren, J. B. Bell, P. Colella, L. H. Howell, and M. L. Welcome. A conservative adaptive projection method for the variable density incompressible Navier-Stokes equations. J. Comput. Phys., 142(1):1-46, 1998.

[BF91] F. Brezzi and M. Fortin. Mixed and hybrid finite element methods. Springer-Verlag, New York, 1991.

[BF06] F. Boyer and P. Fabrie. Eléments d'analyse pour l'étude de quelques modèles d'écoulements de fluides visqueux incompressibles, volume 52 of Mathématiques \& Applications. Springer-Verlag, Berlin, 2006.

[BGP05] D. Bouche, J.-M. Ghidaglia, and F. Pascal. Error estimate and the geometric corrector for the upwind finite volume method applied to the linear advection equation. SIAM J. Numer. Anal., 43(2):578-603 (electronic), 2005.

[BM92] J. B. Bell and D. L. Marcus. A second order projection method for variable-density flows. J. Comput. Phys., 101:334-348, 1992.

[BR96] C.H. Bruneau and P. Rasetarinera. A finite volume method with efficient limiters for solving conservation laws. Internal report 96024, MAB Laboratory, Bordeaux 1 University, France, 1996.

[Cal96] C. Calgaro. Méthodes multi-résolution auto-adaptatives en éléments finis : application aux équations de la mécanique des fluides. PhD thesis, University of Paris-11, Orsay, France, 1996.

[CCGS] C. Calgaro, E. Creusé, Th. Goudon, and C. Schmeiser. Numerical simulations of Low Mach models by using hybrid FV-FE methods. Work in progress.

[CDJ06] C. Calgaro, P. Deuring, and D. Jennequin. A preconditioner for generalized saddlepoint problems: Application to 3d stationary Navier-Stokes equations. Numer. Methods Partial Differential Equations, 22(2):1289-1313, 2006.

[Cha81] S. Chandrasekhar. Hydrodynamic and Hydromagnetic Stability. Dover, 1981. 
[Che04] J.-Y. Chemin. Le système de Navier-Stokes incompressible soixante dix ans après Jean Leray. In Actes des Journées Mathématiques à la Mémoire de Jean Leray, volume 9 of Sémin. Congr., pages 99-123. Soc. Math. France, Paris, 2004.

[Cho68] A.J. Chorin. Numerical solution of the Navier-Stokes equations. Math. Comp., 22:745$762,1968$.

[Dan04] R. Danchin. Local and global well-posedness results for flows of inhomogeneous viscous fluids. Adv. Differential Equations, 9(3-4):353-386, 2004.

[Des04a] B. Després. An explicit a priori estimate for a finite volume approximation of linear advection on non-cartesian grids. SIAM J. Numer. Anal., 42(2):484-504, 2004.

[Des04b] B. Després. Lax theorem and finite volume schemes. Math. Comput., 73(247):1203-1234, 2004.

[DGS87] A. Desideri, A. Goudjo, and V. Selmin. Third-order numerical schemes for hyperbolic problems. Research report INRIA, 607, 1987.

[EG04] A. Ern and J.-L. Guermond. Theory and practice of finite elements, volume 159 of Applied Mathematical Sciences. Springer-Verlag, New York, 2004.

[EGH00] R. Eymard, T. Gallouët, and R. Herbin. Finite volume methods. In Handbook of numerical analysis, Vol. VII, Handb. Numer. Anal., VII, pages 713-1020. North-Holland, Amsterdam, 2000.

[FGQ01] Y. Fraigneau, J.-L. Guermond, and L. Quartapelle. Approximation of variable density incompressible flows by means of finite elements and finite volumes. Commun. Numer. Methods Eng., 17:893, 2001.

[GMS06] J. L. Guermond, P. Minev, and J. Shen. An overview of projection methods for incompressible flows. Comput. Methods Appl. Mech. Engrg., 195(44-47):6011-6045, 2006.

[GP99] J.-M. Ghidaglia and F. Pascal. Passerelles volumes finis-éléments finis. C. R. Acad. Sci. Paris Sér. I Math., 328(8):711-716, 1999.

[GQ00] J.-L. Guermond and L. Quartapelle. A projection FEM for variable density incompressible flows. J. Comput. Phys., 165(1):167-188, 2000.

[GR86] V. Girault and P.-A. Raviart. Finite element methods for Navier-Stokes equations, volume 5 of Springer Series in Computational Mathematics. Springer-Verlag, Berlin, 1986. Theory and algorithms.

[GS89] J. W. Goodrich and W. Y. Soh. Time-dependent viscous incompressible Navier-Stokes equations: the finite difference Galerkin formulation and streamfunction algorithms. $J$. Comput. Phys., 84(1):207-241, 1989. 
[GST04] I. Gasser, J. Struckmeier, and I. Teleaga. Modelling and simulation of fires in vehicle tunnels. Internat. J. Numer. Methods Fluids, 44(3):277-296, 2004.

[Gue99] J. L. Guermond. Stabilization of galerkin approximations of transport equations by subgrid modeling. Modél. Math. Anal. Numér. (M2AN), 33(6):1293-1316, 1999.

[IT99] S. Itoh and A. Tani. Solvability of nonstationary problems for nonhomogeneous incompressible fluids and the convergence with vanishing viscosity. Tokyo J. Math., 22(1):1742, 1999 .

[JL04] H. Johnston and J.-G. Liu. Accurate, stable and efficient Navier-Stokes solvers based on explicit treatment of the pressure term. J. Comput. Phys., 199(1):221-259, 2004.

[Ler34] J. Leray. Sur le mouvement d'un liquide visqueux emplissant l'espace. Acta Math., 63:193-248, 1934.

[LeV02] R. J. LeVeque. Finite volume methods for hyperbolic problems. Cambridge Texts in Applied Mathematics. Cambridge University Press, Cambridge, 2002.

[Lio96] P.-L. Lions. Mathematical topics in fluid mechanics. Vol. 1: Incompressible models, volume 3 of Oxford Lecture Series in Mathematics and its Applications. The Clarendon Press Oxford University Press, New York, 1996. Oxford Science Publications.

[Lio98] P.-L. Lions. Mathematical topics in fluid mechanics. Vol. 2 : Compressible models, volume 10 of Oxford Lecture Series in Mathematics and its Applications. The Clarendon Press Oxford University Press, New York, 1998. Oxford Science Publications.

[MV] S. Martin and J. Vovelle. Convergence of the finite volume method for scalar conservation laws with discontinuous flux function. Modél. Math. Anal. Numér. (M2AN), to appear.

[MV07] B. Merlet and J. Vovelle. Error estimate for finite volume scheme. Numer. Math., 106(1):129-155, 2007.

[Pas03] F. Pascal. On combining finite element methods and finite volume methods in computational fluid dynamics. In Progress in analysis, Vol. I, II (Berlin, 2001), pages 1205-1213. World Sci. Publ., River Edge, NJ, 2003.

[PG01] F. Pascal and J.-M. Ghidaglia. Footbridge between finite volumes and finite elements with applications to CFD. Internat. J. Numer. Methods Fluids, 37(8):951-986, 2001.

[PS07] J. Pyo and J. Shen. Gauge-Uzawa methods for incompressible flows with variable density. J. Comput. Phys., 221(1):181-197, 2007.

[SBJK99] T. Schneider, N. Botta, Geratz K. J., and R. Klein. Extension of finite volume compressible flow solvers to multidimensional, variable density zero Mach number flows. $J$. Comput. Phys., 155:248-286, 1999. 
[SD05a] E. Shapiro and D. Drikakis. Artificial compressibility, characteristics-based schemes for variable density, incompressible, multi-species flows. I. Derivation of different formulations and constant density limit. J. Comput. Phys., 210(2):584-607, 2005.

[SD05b] E. Shapiro and D. Drikakis. Artificial compressibility, characteristics-based schemes for variable-density, incompressible, multispecies flows. II. Multigrid implementation and numerical tests. J. Comput. Phys., 210(2):608-631, 2005.

[SD06] E. Shapiro and D. Drikakis. Non-conservative and conservative formulations of characteristics-based numerical reconstructions for incompressible flows. Internat. J. Numer. Methods Engrg., 66(9):1466-1482, 2006.

[SG88] W. Y. Soh and J. W. Goodrich. Unsteady solution of incompressible Navier-Stokes equations. J. Comput. Phys., 79(1):113-134, 1988.

[Str68] G. Strang. On the construction and comparison of difference schemes. SIAM J. Numer. Anal., 5:506-517, 1968.

[Tem68] R. Temam. Une méthode d'approximation de la solution des équations de Navier-Stokes. Bull. Soc. Math. France, 98:115-152, 1968.

[Tem01] R. Temam. Navier-Stokes equations. AMS Chelsea Publishing, Providence, RI, 2001. Theory and numerical analysis, Reprint of the 1984 edition.

[Try88] G. Tryggvason. Numerical simulations of the Rayleigh-Taylor instability. J. Comput. Phys., 75:235-282, 1988.

[TSG $\left.{ }^{+} 06\right]$ I. Teleaga, M. Seaïd, I. Gasser, A. Klar, and J. Struckmeier. Radiation models for thermal flows at low Mach number. J. Comput. Phys., 215(2):506-525, 2006.

[Tur99] S. Turek. Efficient Solvers for Incompressible Flow Problems. Springer, Lect. Notes in Comput. Sc. and Eng., 1999.

[VL79] B. Van Leer. Towards the ultimate conservative difference scheme v.a. second-order sequel to Godunov's method. J. Comput. Phys., 32:101-136, 1979.

[VL84] B. Van Leer. On the relation between the upwind-diferencing schemes of Godunov, Engquist-Osher and Roe. SIAM J. Sci. Stat. Comput., 5(1):1-20, 1984.

[VM96] V. Venkatakrishnan and D.J. Mavriplis. Implicit method for the computation of unsteady flows on unstructured grids. J. Comput. Phys., 127:380-397, 1996. 